\title{
A GENERALIZED KOSZUL COMPLEX. II. DEPTH AND MULTIPLICITY
}

\author{
BY \\ DAVID A. BUCHSBAUM AND DOCK S. RIM (1)
}

Introduction. In a previous paper [3], a general complex was described which could be associated with a map of modules over a commutative ring. Also mentioned in that paper were several areas which could be investigated in which this complex would play a relevant role. In this paper, we investigate two of those areas, namely depth and multiplicity.

The notion of the $I$-depth of a module $E$, where $I$ is an ideal in a commutative, noetherian ring $R$, was introduced in $[1 ; 7]$. If $f: R^{m} \rightarrow R^{n}$ is a map, then we may consider the annihilator of Coker $\bigwedge^{n} f$ (where $\bigwedge^{k}$ denotes $k$ th exterior product), and call this ideal $I(f)$. We then ask how the $I(f)$-depth of a module may be determined and show that this number may be computed in terms of the homology groups of the Koszul complex associated with the map $f$ (Theorem 2.4). As a result, we obtain another proof of the generalized Cohen-Macaulay unmixedness theorem.

As for multiplicity, the idea is to assign a non-negative integer to a module $M$ of finite length, which agrees with the usual multiplicity when $M$ is of the form $R / \mathfrak{q}$. We do this by studying a generalized Hilbert characteristic polynomial for the module $M$, which involves representing $M$ as the cokernel of a map $f: R^{m} \rightarrow R^{n}$. The study of this polynomial leads to a generalization of the Krull principal ideal theorem(Theorem 3.5). We eventually prove that the Euler-Poincaré characteristic of the complex associated with the map $f: R^{m} \rightarrow R^{n}$ is related to the leading coefficient of the Hilbert polynomial associated with this map (Theorem 4.2).

$\S 1$ deals with the complex associated with a map $f: A \rightarrow B$. Although the definition here differs slightly from that given in [3], it boils down to the same thing when $A=R^{m}$ and $B=R^{n}$. All the properties of this complex which are needed in the subsequent sections of this paper are proved in the first section.

The second section deals with the notion of depth, yielding the generalized Cohen-Macaulay theorem, while the third section treats in detail the characteristic polynomial of a map, the consequent generalization of the Krull principal ideal theorem, and multiplicity of modules of finite length. In this section, we also introduce the idea of a parameter matrix, generalizing the notion of system of

Received by the editors December 11, 1962.

(1) This work was done with the partial support of NSF Grant G-14097 and also with the partial support of Institute for Defense Analyses 
parameters. In the last section we construct a double complex which yields the relationship between the Euler-Poincaré characteristic of the Koszul complex of a map and the leading coefficient of the Hilbert polynomial of the map. The double complex is constructed in far more generality than is needed for this paper, but it will be used in subsequent papers in this series.

It will be evident to the reader that the results obtained in this paper are preliminary in nature. However, they give sufficient indication of the directions in which one may go.

Throughout this paper, all rings will be assumed commutative and noetherian, and all modules will be finitely generated, unless otherwise stated.

1. Complexes associated with a bilinear form. All modules in this section are modules over a fixed ring $R$.

If $\gamma: A \times B \rightarrow R$ is a bilinear form of $R$-modules, each element $a$ in $A$ induces a linear functional on $B$ and in turn is uniquely extended to a derivation $\omega_{a}$ of degree -1 on the exterior algebra, $\wedge B$, of $B$. Since $\omega_{a_{1}} \omega_{a_{2}}(\beta)=-\omega_{a_{2}} \omega_{a_{1}}(\beta)$, we define, for each $\alpha=a_{1} \wedge \cdots \wedge a_{p}$ in $\wedge^{p} A$ and $\beta$ in $\wedge^{q} B, \omega_{\alpha}(\beta)=\omega_{a_{1}} \omega_{a_{2}} \cdots \omega_{a_{p}}(\beta)$ which is an element in $\Lambda^{q-p} B$. Extending by linearity, we obtain a map $\wedge^{p} A \otimes \wedge^{q} B \rightarrow \wedge^{q-p} B$. If $\beta=b_{1} \wedge \cdots \wedge b_{q}$, it is easy to see that

$$
\omega_{\alpha}(\beta)=\sum_{j_{1}<\ldots<j}(-1)^{\Sigma j_{k}} \operatorname{det}\left(\gamma\left(a_{i}, b_{j_{k}}\right)\right) b_{1} \wedge \cdots \wedge \hat{b}_{j_{1}} \wedge \cdots \wedge \hat{b}_{j_{p}} \wedge \cdots \wedge b_{q},
$$

where $\hat{b}$ means to delete $b$.

We define a complex $\mathfrak{C}(\gamma, \wedge A)$ by

$$
\begin{aligned}
\cdots & \rightarrow \wedge B \otimes \overline{\wedge B} \otimes \overline{\wedge B} \otimes \overline{\wedge B} \otimes \wedge A \rightarrow \wedge B \otimes \overline{\wedge B} \otimes \overline{\wedge B} \otimes \wedge A \\
& \rightarrow \wedge B \otimes \overline{\wedge B} \otimes \wedge A \rightarrow \wedge B \otimes \wedge A \rightarrow \wedge A,
\end{aligned}
$$

where $\overline{\wedge B}=\Sigma_{s \geqq 1} \wedge^{s} B$ and the boundary operator $d_{n}$ is defined by

$$
\begin{aligned}
d_{n}\left(\lambda_{0} \otimes \lambda_{1} \otimes \cdots \otimes \lambda_{n-1} \otimes \alpha\right)= & \sum_{i=0}^{n-2}(-1)^{i} \lambda_{0} \otimes \cdots \otimes \lambda_{i} \wedge \lambda_{i+1} \otimes \cdots \otimes \lambda_{n-1} \otimes \alpha \\
& +(-1)^{n-1} \lambda_{0} \otimes \cdots \otimes \lambda_{n-2} \otimes \omega_{-1}(\alpha) .
\end{aligned}
$$

Thus the boundary operator $d$ can be given by the recursion formula:

$$
\begin{aligned}
d_{1}\left(\lambda_{0} \otimes \alpha\right) & =\omega_{\lambda_{0}}(\alpha) \\
d_{n}\left(\lambda_{0} \otimes \lambda_{1} \otimes \beta\right) & =\lambda_{0} \wedge \lambda_{1} \otimes \beta-\lambda_{0} \otimes d_{n-1}\left(\lambda_{1} \otimes \beta\right) .
\end{aligned}
$$

The following lemma shows that $\mathscr{C}(\gamma, \wedge A)$, with the boundary operator $d$ defined above, is a chain complex.

LEMMA 1.1. $d^{2}=0$. 
Proof. $d_{1} d_{2}\left(\lambda_{0} \otimes \lambda_{1} \otimes \alpha\right)=d_{1}\left(\lambda_{0} \wedge \lambda_{1} \otimes \alpha-\lambda_{0} \otimes \omega_{\lambda_{1}}(\alpha)\right)=\omega_{\lambda_{0} \wedge \lambda_{1}}(\alpha)-\omega_{\lambda_{0}} \omega_{\lambda_{1}}(\alpha)=0$. Now, proceeding by induction on $n$, we have

$$
\begin{aligned}
& d_{n} d_{n+1}\left(\lambda_{0} \otimes \lambda_{1} \otimes \lambda_{2} \otimes \beta\right) \\
&= d_{n}\left(\lambda_{0} \wedge \lambda_{1} \otimes \lambda_{2} \otimes \beta-\lambda_{0} \otimes d_{n}\left(\lambda_{1} \otimes \lambda_{2} \otimes \beta\right)\right) \\
&= d_{n}\left(\lambda_{0} \wedge \lambda_{1} \otimes \lambda_{2} \otimes \beta-\lambda_{0} \otimes \lambda_{1} \wedge \lambda_{2} \otimes \beta+\lambda_{0} \otimes \lambda_{1} \otimes d_{n-1}\left(\lambda_{2} \otimes \beta\right)\right) \\
&= \lambda_{0} \wedge \lambda_{1} \wedge \lambda_{2} \otimes \beta-\lambda_{0} \wedge \lambda_{1} \otimes d_{n-1}\left(\lambda_{2} \otimes \beta\right) \\
&-\lambda_{0} \wedge \lambda_{1} \wedge \lambda_{2} \otimes \beta+\lambda_{0} \otimes d_{n-1}\left(\lambda_{1} \wedge \lambda_{2} \otimes \beta\right) \\
&+\lambda_{0} \wedge \lambda_{1} \otimes d_{n-1}\left(\lambda_{2} \otimes \beta\right)-\lambda_{0} \otimes d_{n-1}\left(\lambda_{1} \otimes d_{n-1}\left(\lambda_{2} \otimes \beta\right)\right) \\
&= \lambda_{0} \otimes d_{n-1} d_{n}\left(\lambda_{1} \otimes \lambda_{2} \otimes \beta\right)=0(\text { by induction hypothesis }) .
\end{aligned}
$$

It is clear that the correspondence $(\gamma ; A, B) n \rightarrow \mathbb{C}(\gamma, \wedge A)$ or $(\gamma ; A, B)$ $n \rightarrow \mathfrak{C}(\gamma, B)$ is a functor from the category of bilinear forms into the category of chain complexes, where the category of bilinear forms has as its objects the forms $(\gamma ; A, B)$, and as maps $(\gamma ; A, B) \rightarrow\left(\gamma^{\prime} ; A^{\prime}, B^{\prime}\right)$, pairs of maps $f_{A}: A \rightarrow A^{\prime}$, $f_{B}: B \rightarrow B^{\prime}$ such that $\gamma^{\prime}\left(f_{A} \otimes f_{B}\right)=\gamma$.

In this paper, we will be interested in certain subcomplexes of the complex $\mathfrak{C}(\gamma, \wedge A)$. Namely, for each pair of integers $p, q$, we define the complex $\mathfrak{C}\left(\gamma, \wedge^{p} A, q\right)$ by

$$
\begin{aligned}
\cdots & \rightarrow \sum_{s_{0} \geqq q} \wedge^{s_{0}} B \otimes \wedge^{s_{1}} B \otimes \wedge^{s_{2}} B \otimes \wedge^{p+\Sigma s_{i}} A \rightarrow \sum_{s_{0} \geqq q} \wedge^{s_{0}} B \otimes \wedge^{s_{1}} B \otimes \wedge^{p+\Sigma s_{i}} A \\
& \rightarrow \sum_{s_{0} \geqq q} \wedge^{s_{0}} B \otimes \wedge^{p+s_{0}} A \rightarrow \wedge^{p} A,
\end{aligned}
$$

where $s_{i} \geqq 1$ for $i>0$. Similarly, $\mathfrak{C}\left(\gamma, \wedge^{p} B, q\right)$ can be defined.

Our notation $\mathbb{C}\left(\gamma, \wedge^{p} A, q\right)$ may be a little ambiguous since

$$
\mathfrak{C}\left(\gamma, \wedge^{-1} A, q\right) \neq \mathfrak{C}\left(\gamma, \wedge^{-2} A, q\right)
$$

whereas $\wedge^{-1} A=\wedge^{-2} A=0$. However, we use this notation to specify the degree $p$, as well as the module $A$.

It is clear that a map $(\gamma ; A ; B) \rightarrow\left(\gamma^{\prime} ; A^{\prime}, B^{\prime}\right)$ of bilinear forms induces a chain map $\mathfrak{C}\left(\gamma, \wedge^{p} A, q\right) \rightarrow \mathfrak{C}\left(\gamma^{\prime}, \wedge^{p} A^{\prime}, q\right)$, i.e., $(\gamma ; A, B) \sim \rightarrow \mathfrak{C}\left(\gamma, \wedge^{p} A, q\right)$ is a functor.

Proposition 1.2. Given a bilinear form $\gamma: A \times B \rightarrow R$, we have:

(1) $\mathfrak{C}\left(\gamma, \wedge^{p} A, q\right)$ is homotopically trivial for $q \leqq 0$.

(2) $0 \rightarrow \mathfrak{C}\left(\gamma, \wedge^{p} A, q+1\right) \rightarrow \mathfrak{C}\left(\gamma, \wedge^{p} A, q\right) \rightarrow \wedge^{p} B \otimes \mathfrak{C}\left(\gamma, \wedge^{p+q} A, 1\right) \rightarrow 0$ is exact.

(3) If $A=A^{\prime} \oplus R$, then $0 \rightarrow \mathfrak{C}\left(\gamma^{\prime}, \wedge^{p} A^{\prime}, q\right) \rightarrow \mathfrak{C}\left(\gamma, \wedge^{p} A, q\right) \rightarrow \mathbb{C}\left(\gamma^{\prime}, \wedge^{p-1} A^{\prime}, q\right) \rightarrow 0$ is exact where $\gamma^{\prime}$ is the restriction of $\gamma$ to $A^{\prime} \times B$.

Proof. (1) Define a homotopy $s: \mathbb{C}_{n}\left(\gamma, \wedge^{p} A, q\right) \rightarrow \mathbb{C}_{n+1}\left(\gamma, \wedge^{p} A, q\right)$ by $s(\alpha)=1 \otimes \alpha$. Then $(d s+s d)(\alpha)=d(1 \otimes \alpha)+1 \otimes d(\alpha)=\alpha-1 \otimes d(\alpha)+1 \otimes d(\alpha)=\alpha$. 
(2) $\mathfrak{C}\left(\gamma, \wedge^{p} A, q+1\right)$ is a subcomplex of $\mathfrak{C}\left(\gamma, \wedge^{p} A, q\right)$ and $\mathfrak{C}\left(\gamma, \wedge^{p} A, q\right)$ modulo $\mathfrak{C}\left(\gamma, \wedge^{p} A, q+1\right)$ is simply $\wedge^{q} B \otimes \mathfrak{C}\left(\gamma, \wedge^{p+q} A, 1\right)$. We observe here that the canonical map $\mathfrak{C}\left(\gamma, \wedge^{p} A, q\right) \rightarrow \wedge^{q} B \otimes \mathfrak{C}\left(\gamma, \wedge^{p+q} A, 1\right)$ is of degree -1 , i.e.,

$$
0 \rightarrow \mathfrak{C}_{n}\left(\gamma, \wedge^{p} A, q+1\right) \rightarrow \mathfrak{C}_{n}\left(\gamma, \wedge^{p} A, q\right) \rightarrow \wedge^{q} B \otimes \mathfrak{C}_{n-1}\left(\gamma, \wedge^{p+q} A, 1\right) \rightarrow 0
$$

is exact.

(3) $\mathfrak{C}\left(\gamma^{\prime}, \wedge^{p} A^{\prime}, q\right)$ is a subcomplex of $\mathfrak{C}\left(\gamma, \wedge^{p} A, q\right)$ and, under the identification $\wedge^{s} A=\wedge^{s} A^{\prime} \oplus^{s-1} A^{\prime}, \mathbb{C}\left(\gamma, \wedge^{p} A, q\right)$ modulo $\mathbb{C}\left(\gamma^{\prime}, \wedge^{p} A^{\prime}, q\right)$ is simply $\mathfrak{C}\left(\gamma, \wedge^{p-1} A^{\prime}, q\right)$.

Corollary 1.3. Assume that $B$ is $R$-projective. Then the following statements are equivalent:

(1) $\mathfrak{C}\left(\gamma, \wedge^{p} A, q\right)$ is acyclic for all $p, q$ such that $p+q=n$.

(2) $\mathfrak{C}\left(\gamma, \wedge^{\cdot} A, q\right)$ is acyclic for all $p, q$ such that $p+q \leqq n$.

Proof. (1) $\Rightarrow(2)$ : It suffices to show that the acyclicity of $\mathbb{C}\left(\gamma, \wedge^{p} A, m-p\right)$ for all $p$ implies the acyclicity of $\mathfrak{C}\left(\gamma, \wedge^{p} A, m-p-1\right)$ for all $p$, where $m$ is an arbitrary integer. But this follows immediately from the exactness (by 1.2, (2)) of $0 \rightarrow \mathfrak{C}\left(\gamma \wedge^{p} A, m-p\right) \rightarrow \mathfrak{C}\left(\gamma, \wedge^{p} A, m-p-1\right) \rightarrow \wedge^{m-p-1} B \otimes C\left(\gamma, \wedge^{m-1} A, 1\right) \rightarrow 0$, together with the fact that $B$, and hence also $\wedge^{m-p-1} B$, is projective.

Given an $R$-homomorphism $f: R^{m} \rightarrow R^{n}$, we have a bilinear form

$$
\gamma(f): R^{m} \times R^{n *} \rightarrow R, \text { where } R^{n *}=\operatorname{Hom}\left(R^{n}, R\right),
$$

and in turn we get the complexes $\mathfrak{C}\left(\gamma(f), \wedge^{p} R^{m}, q\right)$. We define the (generalized) Koszul complex, $K\left(\wedge^{p} f\right)$, for each $p$ to be the complex $\mathfrak{C}\left(\gamma(f), \wedge^{p} R^{m}, n+1-p\right)$ augmented by the map

$$
\begin{aligned}
& \wedge^{p} R^{m} \stackrel{\wedge^{p} f}{\longrightarrow} \wedge^{p} R^{n}, \\
& \text { i.e., } \\
& \begin{aligned}
\cdots & \rightarrow \sum_{s_{0} \geqq n+1-p} \wedge^{s_{0}} R^{n *} \otimes \Lambda^{s_{1}} R^{n *} \otimes \Lambda^{p+\Sigma s_{i}} R^{m} \rightarrow \sum_{s_{0} \geqq n+1-p} \wedge^{s_{0}} R^{n *} \otimes \wedge^{p+s_{0}} R^{m} \\
& \stackrel{d}{\rightarrow} \Lambda^{p} R^{m} \stackrel{\Lambda^{p} f}{\longrightarrow} \wedge^{p} R^{n}
\end{aligned}
\end{aligned}
$$

where $s_{i} \geqq 1$ for all $i \geqq 1$. It is easy to see that $\wedge^{p} f \cdot d=0$ and $K\left(\bigwedge^{p} f\right)$ is a chain complex of length $m-n+1$ for $1 \leqq p \leqq n$. This Koszul complex, $K\left(\wedge^{p} f\right)$, may be viewed as an approximation to a projective resolution of the $R$-module, Coker $\wedge^{p} f$.

For each $R$-module $E, H_{*}\left(\wedge^{p} f, E\right)$ and $H^{*}\left(\wedge^{p} f, E\right)$ shall mean the homology and cohomology of the complex $K\left(\bigwedge^{p} f\right)$ with coefficients in $E$, i.e., $H^{*}\left(\wedge^{p} f, E\right)$ $=H\left(K\left(\bigwedge^{p} f\right) \otimes E\right)$ and $H^{*}\left(\bigwedge^{p} f, E\right)=H\left(\operatorname{Hom}\left(K\left(\wedge^{p} f\right), E\right)\right)$.

Proposition 1.4. (1) For any map $f: R^{m} \rightarrow R^{n}, K\left(\bigwedge^{p} f\right)$ is homotopically trivial whenever $p>n$ or $p \leqq 0$. 
(2) If $f: R^{m} \rightarrow R^{n}$ is an epimorphism, then $\mathbb{C}\left(\gamma(f), \wedge^{p} R^{m}, q\right)$ is homotopically trivial for all pairs $p, q$ such that $p+q \leqq n$.

(3) If $f: R^{m} \rightarrow R^{n}$ is an epimorphism, then $K\left(\wedge^{p} f\right)$ is homotopically trivial for all $p$.

Proof. (1) If $p>n$, then $\wedge^{p} R^{n}=0$ and hence $K\left(\wedge^{p} f\right)=\mathfrak{C}\left(\gamma(f), \bigwedge^{p} R^{m}, n+1-p\right)$. But then, by (1) of 1.2 , it is homotopically trivial since $n+1-p \leqq 0$. The case $p \leqq 0$ is trivial to see.

(2) Since $\mathbb{C}\left(\gamma(f), \wedge^{p} R^{m}, q\right)$ is a free complex, homotopic triviality is the same as acyclicity. By 1.3 , it suffices then to show that $\mathscr{C}\left(\gamma(f), \wedge^{p} R^{m}, n-p\right)$ is acyclic for all $p$. If, however, we can show acyclicity after localizing at each maximal ideal of $R$, we will have this result. Hence we may assume that $R$ has a unique maximal ideal, and we proceed by induction on $m$.

If $m=n$ (since $f$ is assumed to be an epimorphism, we of course have $m \geqq n$ ), then $f: R^{m} \rightarrow R^{n}$ must be an isomorphism, since it is onto. The complex $\mathfrak{C}\left(\gamma(f), \wedge^{p} R^{n}, n-p\right)$ is simply $\wedge^{n-p} R^{n *} \otimes \wedge^{n} R^{n} \rightarrow \wedge^{p} R^{n}$, which is obviously an isomorphism. Thus for $m=n$, we have the result. If $m>n$, we can always find a decomposition $R^{m}=R^{m-1} \oplus R$ such that $f$ restricted to $R^{m-1}$ is still an epimorphism (since projectives over $R$ are free). Letting $f^{\prime}$ denote this restriction of $f$ to $R^{m-1}$, we obtain the exact sequence

$0 \rightarrow \mathbb{C}\left(\gamma\left(f^{\prime}\right), \wedge^{p} R^{m-1}, n-p\right) \rightarrow \mathbb{C}\left(\gamma(f), \wedge^{p} R^{m}, n-p\right) \rightarrow \mathbb{C}\left(\gamma\left(f^{\prime}\right), \wedge^{p-1} R^{m-1}, n-p\right) \rightarrow 0$.

Since the induction hypothesis implies the acyclicity of the extreme complexes, we obtain the acyclicity of the middle one.

(3) We again proceed by induction on $m$, the case $m=n$ being trivial. We first observe that the exact sequence in (3) of 1.2 , augmented by $\wedge^{p} R^{n}$, takes the form

$$
0 \rightarrow K\left(\wedge^{p} f^{\prime}\right) \rightarrow K\left(\wedge^{p} f\right) \rightarrow \mathscr{C}\left(\gamma\left(f^{\prime}\right), \wedge^{p-1} R^{m-1}, n+1-p\right) \rightarrow 0,
$$

where $f^{\prime}$ is again the restriction of $f$ to $R^{m-1}$. If we choose the summand $R^{m-1}$ of $R^{m}$ so that $f^{\prime}$ is still an epimorphism, then the induction hypothesis yields the acyclicity of $K\left(\wedge^{p} f^{\prime}\right)$, and part (2) of this proposition gives the acyclicity of the right-hand complex (since $p-1+n+1-p=n$ ). Thus the middle complex, $K\left(\wedge^{p} f\right)$, is acyclic. Since $K\left(\bigwedge^{p} f\right)$ is also a free complex, acyclicity implies homotopic triviality.

Corollary 1.5( $\left(^{2}\right)$. Supp $H_{*}\left(\wedge^{p} f, E\right)$ and Supp $H^{*}\left(\wedge^{p} f, E\right)$ are both contained in Supp Coker $f \cap$ Supp $E$.

Proof. It is clear that for any prime ideal $\mathfrak{p}$ in $R$, the localization $\left(K\left(\wedge^{p} f\right)\right)_{\mathfrak{p}}$ is the same as $K\left(\wedge^{p} f \otimes R_{\mathfrak{p}}\right)$. Thus, if $\mathfrak{p}$ is not in Supp Coker $f$, then $f \otimes R_{\mathfrak{p}}$ is an

(2) For each $R$-module $M$, Supp $M=$ the set of all prime ideals $\mathfrak{p}$ in $R$ such that $M_{\mathfrak{p}} \neq 0$. It follows from primary decomposition theory that Supp $M=$ the set of all primes containing Ann $(M)$ where $\operatorname{Ann}(M)$ is the set of all $r \in R$ such that $r M=0$. 
epimorphism and so the complex $\left(K\left(\Lambda^{p} f\right)\right)_{\mathfrak{p}}$ is homotopically trivial; thus $\mathfrak{p}$ is neither in Supp $H_{*}\left(\wedge^{p} f, E\right)$ nor in Supp $H^{*}\left(\wedge^{p} f, E\right)$. Of course, if $\mathfrak{p}$ is not in Supp $E$, the conclusion is even more obvious.

We will now give another description of the complex $\mathscr{C}\left(\gamma(f), \wedge{ }^{p} R^{m}, q\right)$ which will be used later.

If we are given a map $g: A \rightarrow B$ of $R$-modules, then we get the algebra map $\wedge g: \wedge A \rightarrow \wedge B$, and in turn we get the complex $\mathfrak{D}(g, \wedge B)$ which is defined by

$$
\cdots \rightarrow \wedge A \otimes \overline{\wedge A} \otimes \overline{\wedge A} \otimes \wedge B \rightarrow \wedge A \otimes \overline{\wedge A} \otimes \wedge B \rightarrow \wedge A \otimes \wedge B \rightarrow \wedge B,
$$

where $\overline{\wedge A}=\Sigma_{s \geqq 1} \wedge^{s} A$ and

$$
\begin{aligned}
d\left(\lambda_{0} \otimes \lambda_{1} \otimes \cdots \otimes \lambda_{p} \otimes \beta\right)= & \sum_{i=0}^{p-1}(-1) \lambda_{0} \otimes \cdots \otimes \lambda_{i} \wedge \lambda_{i+1} \otimes \cdots \otimes \lambda_{p} \otimes \beta \\
& +(-1)^{p} \lambda_{0} \otimes \cdots \otimes \lambda_{p-1} \otimes \lambda_{p} \wedge \beta
\end{aligned}
$$

$\left(\lambda_{p} \wedge \beta\right.$ of course means $\left.\wedge g\left(\lambda_{p}\right) \wedge \beta\right)$.

For each pair of integers $p$ and $q$, we may consider the subcomplex $\mathfrak{D}\left(g, \wedge^{p} B, q\right)$ of $\mathfrak{D}(g, \wedge B)$, defined by

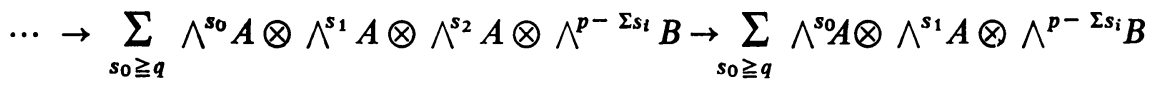

$$
\begin{aligned}
& \rightarrow \sum_{s_{0} \geqq q} \wedge^{s_{0}} A \otimes \wedge^{p-s_{0}} B \rightarrow \wedge^{p} B
\end{aligned}
$$

where $s_{i} \geqq 1$ for $i \geqq 1$.

Now let a map $f: R^{m} \rightarrow R^{n}$ be given. Then it induces $f^{*}: R^{n *} \rightarrow R^{m *}$ and so we may consider the complex $\mathfrak{D}\left(f^{*}, \wedge^{p} R^{m *}, q\right)$. We have

PROPOSITION 1.6. $\mathscr{C}\left(\gamma(f), \wedge^{p} R^{m}, q\right) \approx \mathfrak{D}\left(f^{*}, \wedge^{m-p} R^{m *}, q\right)$ as chain complexes.

Proof. Let $y_{1}, \cdots, y_{m}$ be a free basis of $R^{m}$, and set $\xi=y_{1}^{*} \wedge \cdots \wedge y_{m}^{*}$ which is a generator of $\bigwedge^{m} R^{m *}\left(y_{1}^{*}, \cdots, y_{m}^{*}\right.$ is the dual basis to $\left.y_{1}, \cdots, y_{m}\right)$. Then $h: \wedge R^{m} \rightarrow \wedge R^{m *}$ given by

$$
h(\alpha)=\sum_{i_{1}<\cdots<i_{t}} \omega_{\xi}\left(\alpha \wedge y_{i_{1}} \wedge \cdots \wedge y_{i_{z}}\right) y_{i_{1}}^{*} \wedge \cdots \wedge y_{i_{t}}^{*}\left(\alpha \in \wedge^{m-t} R^{m}\right)
$$

is an isomorphism, which sends $\wedge^{m-t} R^{m}$ onto $\wedge^{t} R^{m *}$. Looking at the two complexes $\mathfrak{C}\left(\gamma(f), \wedge^{p} R^{m}, q\right)$ :

$$
\begin{aligned}
& \cdots \rightarrow \sum_{s_{0} \geqq q} \bigwedge^{s_{0}} R^{n *} \otimes \bigwedge^{s_{1}} R^{n *} \otimes \bigwedge^{p+\Sigma s_{t}} R^{m} \rightarrow \sum_{s_{0} \geqq q} \wedge^{s_{0}} R^{n *} \otimes \bigwedge^{p+s_{0}} R^{m} \rightarrow \bigwedge^{p} R^{m}, \\
& \mathcal{D}\left(f^{*}, \wedge^{m-p} R^{m *}, q\right) \text { : } \\
& \cdots \rightarrow \sum_{s_{0} \geqq q} \wedge^{s_{0}} R^{n *} \otimes \wedge^{s_{1}} R^{n *} \otimes \wedge^{m-\left(p+\Sigma s_{i}\right)} R^{m *} \rightarrow \sum_{s_{0} \geqq q} \wedge^{s_{0}} R^{n *} \otimes \wedge^{m-\left(p+s_{0}\right)} R^{m *} \\
& \rightarrow \wedge^{m-p} R^{m *}
\end{aligned}
$$


we are led to define a map $\mathfrak{C}\left(\gamma(f), \wedge^{p} R^{m}, q\right) \rightarrow \mathfrak{D}\left(f^{*}, \wedge^{m-p} R^{m *}, q\right)$ by $u_{0} \otimes \cdots \otimes u_{k} \otimes \alpha \rightarrow u_{0} \otimes \cdots \otimes u_{k} \otimes h(\alpha)$, which is an isomorphism of modules. To show that it is a chain isomorphism, it suffices to show that $h \omega_{u}(\alpha)= \pm u h(\alpha)$ for $u$ in $\wedge R^{n *}$ and $\alpha$ in $\wedge R^{m}$. We may, of course, actually assume $u$ is in $\wedge R^{m *}$. We claim that $h \omega_{u}(\alpha)=(-1)^{v(u, \alpha)} u \wedge h(\alpha)$ where

$$
v(u, \alpha)=(d(\alpha)-1)+(d(\alpha)-2)+\cdots+(d(\alpha)-d(u))
$$

where $d(\beta)$ means the homogeneous degree of $\beta$. To show this, we first observe that if $h \omega_{u_{i}}(\alpha)=(-1)^{v\left(u_{i}, \alpha\right)} u_{i} \wedge h(\alpha)$ for all $\alpha \quad(i=1,2)$, then

$$
\begin{aligned}
h \omega_{u_{1} \wedge u_{2}}(\alpha) & =h \omega_{u_{1}} \omega_{u_{2}}(\alpha)=(-1)^{v\left(u_{1}, \omega u_{2}(\alpha)\right)} u_{1} \wedge h \omega_{u_{2}}(\alpha) \\
& =(-1)^{v\left(u_{1} \wedge u_{2}, \alpha\right)} u_{1} \wedge u_{2} \wedge h(\alpha)
\end{aligned}
$$

(since $v\left(u_{1}, \omega_{u_{2}}(\alpha)\right)+v\left(u_{2}, \alpha\right)=\left(d(\alpha)-d\left(u_{2}\right)-1\right)+\cdots+\left(d(\alpha)-d\left(u_{2}\right)-d\left(u_{2}\right)\right)$ $\left.+(d(\alpha)-1)+\cdots+\left(d(\alpha)-d\left(u_{2}\right)\right)=v\left(u_{1} \wedge u_{2}, \alpha\right)\right)$. Therefore we may assume that $u$ is in $R^{m *}$, and because of linearity, we may assume $u=y_{1}^{*}$ and $\alpha=y_{i_{1}} \wedge \cdots \wedge y_{i_{m-t}}$ with $i_{1}<\cdots<i_{m-t}$. If $i_{1} \neq 1$, then it is trivial to see that $h \omega_{u}(\alpha)=0=u \wedge h(\alpha)$. Thus we may assume that $\alpha=y_{1} \wedge \cdots \wedge y_{m-t}$. Then

$$
\begin{aligned}
h \omega y_{1}^{*}\left(y_{1} \wedge \cdots \wedge y_{m-t}\right) & =h\left(y_{2} \wedge \cdots \wedge y_{m-t}\right) \\
& =\Sigma \omega_{\xi}\left(y_{2} \wedge \cdots \wedge y_{m-t} \wedge y_{i_{1}} \wedge \cdots \wedge y_{i_{t+1}}\right) y_{i_{1}}^{*} \wedge \cdots \wedge y_{i_{t+1}}^{*} \\
& =(-1)^{m-t-1} \omega_{\xi}\left(y_{1} \wedge \cdots \wedge y_{m}\right) y_{1}^{*} \wedge y_{m-t+1}^{*} \wedge \cdots \wedge y_{m}^{*} \\
& =(-1)^{m-t-1} y_{1}^{*} \wedge h\left(y_{1} \wedge \cdots \wedge y_{m-t}\right) .
\end{aligned}
$$

Since $m-t-1=v\left(y_{1}^{*}, y_{1} \wedge \cdots \wedge y_{m-t}\right)$, this completes the proof.

2. The depth of a matrix and Koszul complex. For an $R$-module $E$, a sequence of elements $\alpha_{1}, \cdots, \alpha_{d}$ in $R$ is called a proper $E$-sequence if, for each $i, 1 \leqq i \leqq d$, $E /\left(\alpha_{1}, \cdots, \alpha_{i}\right) E \neq 0$ and $\alpha_{i}$ is not a zero divisor for $E /\left(\alpha_{1}, \cdots, \alpha_{i-1}\right) E$. Given an ideal $I$ in $R$ and an $R$-module $E$ such that $R / I \otimes E \neq 0$, the length of a maximal proper $E$-sequence contained in the ideal $I$ is a finite number and does not depend on the choice of a maximal proper $E$-sequence $[1 ; 7]$. We call this length the $I$-depth of $E\left({ }^{3}\right)$ and denote it by depth $(I ; E)$. (It is customary to define depth $(I ; E)=\infty$ if $R / I \otimes E=0$, i.e., if Supp $R / I \cap \operatorname{Supp} E=\varnothing$.) It is well known (and actually is an immediate consequence of our Proposition 2.1 below) that depth $(I ; E)=$ depth $(\operatorname{rad} I ; E)=\inf _{\mathfrak{p} \supset I}$ depth $\left(I_{\mathfrak{p}} ; E_{\mathfrak{p}}\right)$. We further define $\operatorname{depth} E=\inf _{\mathfrak{m}} \operatorname{depth}(\mathfrak{m} ; E)$ where $\mathfrak{m}$ runs through all maximal ideals of $R$. The main purpose of this section is to relate the integer, depth $(I ; E)$, with a homological invariant of the Koszul complex, generalizing the results in [2]. We begin with a proposition of a general nature.

(3) Previously it was called $I$-codimension of $E[1 ; 2]$, but we are adopting Grothendieck's terminology to avoid possible confusion. 
Proposition 2.1. Given an ideal $I$ in $R$ let $T^{i}(i \geqq 0)$ be an exact connected sequence of covariant functors [4] from the category of finitely generated $R$ modules into itself such that

(1) $T(\alpha)=\alpha$ for every homothety $\alpha$ in $R$.

(2) $\operatorname{Supp} T^{i}(E) \subset \operatorname{Supp} R / I$ and $T^{\circ}(E)=0$ if and only if $\operatorname{Supp} R / I \cap A s(E)=\varnothing$ where $A s(E)$ is the set of all associated primes to the $R$-module $E$. Then for each $R$-module $E$ such that $E / I E \neq 0$, we have that depth $(I ; E)=$ the smallest integer $q$ for which $T^{q}(E) \neq 0$. Furthermore, if $T^{0}(*)=\operatorname{Hom}(M, *)$ for some fixed $R$ module $M$, then $T^{d}(E)=\operatorname{Ext}_{R}^{d}(M, E)$, where $d=\operatorname{depth}(I ; E)$.

Proof. Let $c(E)$ be the smallest integer $q$ for which $T^{q}(E) \neq 0$. We show that $c(E)=\operatorname{depth}(I ; E)$ by induction on the non-negative integer $\operatorname{depth}(I ; E)$.

Since $E / I E \neq 0, \operatorname{depth}(I ; E)=0$ if and only if every element in $I$ is a zerodivisor for $E$, i.e., if and only if $I$ is in the union of the associated primes of $E$. Thus $\operatorname{depth}(I ; E)=0$ if and only if $\operatorname{Supp} R / I \cap A s(E) \neq \varnothing$, i.e., if and only if $T^{0}(E) \neq 0$. Hence $\operatorname{depth}(I ; E)=0$ if and only if $c(E)=0$.

Assume now that depth $(I ; E)>0$, and let $\alpha_{1}, \cdots, \alpha_{d}$ be a proper $E$-sequence contained in $I$ with $d=\operatorname{depth}(I ; E)$. Then $0 \rightarrow E \stackrel{\alpha_{1}}{\rightarrow} E \rightarrow E / \alpha_{1} E \rightarrow 0$ is exact and $E / \alpha_{1} E \neq 0$. Furthermore, $\operatorname{depth}\left(\mathrm{I} ; E / \alpha_{1} E\right)=\mathrm{d}-1$ and hence $c\left(E / \alpha_{1} E\right)$ $=\operatorname{depth}\left(I ; E / \alpha_{1} E\right)$ by our induction assumption. It follows from the exactness of

$$
\cdots \rightarrow T^{i-1}\left(E / \alpha_{1} E\right) \rightarrow T^{i}(E) \stackrel{T^{i}\left(\alpha_{1}\right)}{\longrightarrow} T^{i}(E) \rightarrow T^{i}\left(E / \alpha_{1} E\right) \rightarrow \cdots
$$

that $T^{i}\left(\alpha_{1}\right)$ is a monomorphism for all $i \leqq c\left(E / \alpha_{1} E\right)$. Consequently we must have that $T^{i}(E)=0$ for all $i \leqq c\left(E / \alpha_{1} E\right)$ since Supp $T^{i}(E) \subset \operatorname{Supp} R / I$ and hence $T^{i}\left(\alpha_{1}\right)^{h}=T^{i}\left(\alpha_{1}^{h}\right)=\alpha_{1}^{h}$ is in Ann $T^{i}(E)$ for sufficiently large $h$. Furthermore, this implies that $0 \rightarrow T^{c_{1}}\left(E / \alpha_{1} E\right) \rightarrow T^{c_{1}+1}(E)$ is exact, where $c_{1}=c\left(E / \alpha_{1} E\right)$. Thus $T^{c_{1}+1}(E) \neq 0$ and so we may conclude that $c(E)=c_{1}+1=\operatorname{depth}\left(I ; E / \alpha_{1} E\right)+1$ $=$ depth $(I ; E)$. This proves our first statement.

Now the functors $\operatorname{Ext}^{i}(R / I, *)$ and $\operatorname{Ext}^{i}(R / \operatorname{rad}(I), *)$ both satisfy the conditions of our proposition. Thus what we have just proved above yields the fact that $\operatorname{depth}(I ; E)=\operatorname{depth}(\operatorname{rad} I ; E)$.

Let us prove the second statement in the proposition, and thus assume that $T^{0}(*)=\operatorname{Hom}(M, *)$ for some fixed $R$-module $M$. From the fact that $\operatorname{Supp} \operatorname{Hom}(M, R / \mathfrak{p})=\operatorname{Supp} T^{0}(R / \mathfrak{p}) \subset \operatorname{Supp} R / I$, we see that $M_{\mathfrak{p}}=0$ for all $\mathfrak{p}$ not in $\operatorname{Supp} R / I$, i.e., Supp $M$ is contained in $\operatorname{Supp} R / I$. Thus both $T^{i}(*)$ and $\operatorname{Ext}^{i}(M, *)$ are exact connected sequences of functors satisfying the conditions of our proposition.

Now if $\alpha_{1}, \cdots, \alpha_{d}$ is a proper $E$-sequence contained in the annihilator of $T^{d}(E)$, and $d=\operatorname{depth}(I ; E)$ then it is easy to see (since $T^{i}(E)$ is an exact connected sequence of functors) that $T^{\mathrm{o}}\left(E /\left(\alpha_{1}, \cdots, \alpha_{d}\right) E\right)$ is isomorphic to $T^{d}(E)$. If, moreover, $\alpha_{1}, \cdots, \alpha_{d}$ are also contained in the annihilator of $M$, then we have 
$\operatorname{Ext}^{0}\left(M, E /\left(\alpha_{1}, \cdots, \alpha_{d}\right) E\right) \approx \operatorname{Ext}^{d}(M, E)$. Thus, if we can find a proper $E$-sequence $\alpha_{1}, \cdots, \alpha_{d}$ with $\alpha_{i}$ in $\operatorname{Ann}\left(T^{d}(E)\right) \cap \operatorname{Ann}(M)$, we will have

$$
T^{d}(E) \approx T^{0}\left(E /\left(\alpha_{1}, \cdots, \alpha_{d}\right) E\right)=\operatorname{Ext}^{0}\left(M, E /\left(\alpha_{1}, \cdots, \alpha_{d}\right) E\right) \approx \operatorname{Ext}^{d}(M, E),
$$

and our assertion will be proved.

To this end, let $J=\operatorname{Ann}(M) \cap \operatorname{Ann}\left(T^{d}(E)\right) \cap I$. Since Supp $R / I$ contains both Supp $M$ and Supp $T^{d}(E)$, we have that $\operatorname{rad}(J)=\operatorname{rad}(I)$ and hence $\operatorname{depth}(J ; E)$ $=\operatorname{depth}(I ; E)=d$. Therefore we see that we can find $\alpha_{1}, \cdots, \alpha_{d}$ as we wanted, and this completes the proof of our proposition.

What we want to do now is to apply this proposition to considerations of $\mathrm{H}_{*}\left(\wedge^{p} f, E\right)$ and $H^{*}\left(\wedge^{\prime} f, E\right)$, and for this purpose, we need to compute the homology group of highest dimension. To do this, let us first fix some notation.

Given a map $f: R^{m} \rightarrow R^{n}$, we consider the map

$$
k_{q, v}: \wedge^{q+1} R^{n} \otimes S_{v-1}(\text { Coker } f) \rightarrow \wedge^{q} R^{n} \otimes S_{v}(\text { Coker } f)
$$

(where $S(M)=\Sigma_{v \geq 0} S_{v}(M)$ denotes the symmetric algebra generated by $M$ over $R$ ) given by $k_{q, v}\left(x_{1} \wedge \cdots \wedge x_{q+1} \otimes \alpha\right)=\Sigma(-1)^{i} x_{1} \wedge \cdots \wedge \hat{x}_{i} \wedge \cdots \wedge x_{q+1} \otimes x_{i} \alpha$, and we define $\Gamma_{q, v}(f)=$ Coker $k_{q, v}$. The operation of $R^{n}$ on $S($ Coker $f)$ is that obtained through the algebra map $S\left(R^{n}\right) \rightarrow S($ Coker $f)$, observing that $R^{n}=S_{1}\left(R^{n}\right)$.

Lemma 2.2. Supp $\Gamma_{q, v}(f)=$ Supp Coker $f$ for all $v \geqq 1$, and all $q$ such that $1 \leqq q \leqq n$.

Proof. Let $q, v$ be as above. It suffices to show that if Coker $f \neq 0$, then $\Gamma_{q, v}(f) \neq 0$, i.e., $k_{q, v}$ is not onto. For this, we may localize at a prime ideal in Supp Coker $f$, and thus may assume that $R$ is a local ring. Going one step further, we may replace $R$ by its residue field. Thus we are now in the position where $R$ is a field, and $M=$ Coker $f$ is a nonzero vector space over $R$. Let $x_{1}, \cdots, x_{n}$ be a free basis of $R^{n}$ such that $x_{1} \bmod f\left(R^{m}\right)$ is a nonzero element of $M$. Then

$$
k_{q-1, v+1}\left(x_{1} \wedge \cdots \wedge x_{q} \otimes \alpha\right)=\Sigma(-1)^{i} x_{1} \wedge \cdots \wedge \hat{x}_{i} \wedge \cdots \wedge x_{q} \otimes x_{i} \alpha \neq 0
$$

for any $\alpha$ in $S_{v}(M)$ which is not equal to zero, since $x_{2} \wedge \cdots \wedge x_{q} \otimes x_{1} \alpha \neq 0$. Therefore $x_{1} \wedge \cdots \wedge x_{q} \otimes \alpha$ is not in Ker $k_{q-1, v+1}$. But $k_{q-1, v+1} k_{q, v}=0$ implies that $\operatorname{Im} k_{q, v} \subset \operatorname{Ker} k_{q-1, v+1}$ and hence $x_{1} \wedge \cdots \wedge x_{q} \otimes \alpha \notin \operatorname{Im} k_{q, v}$, i.e., $k_{q, v}$ is not onto.

Remark. One may observe that $\Gamma_{1, v}(f)=S_{v+1}($ Coker $f)$ and $\Gamma_{n, v}(f)$ $=S_{v}(\operatorname{Coker} f)$.

Proposition 2.3. Given a map $f: R^{m} \rightarrow R^{n}$, we have

$$
H_{m-n+1}\left(\wedge^{p} f, E\right)= \begin{cases}\operatorname{Hom}\left(\operatorname{Coker} \wedge^{m-p} f, E\right) & \text { if } m=n \text { or } n+1, \\ \operatorname{Hom}\left(\Gamma_{n+1-p, m-n-1}(f), E\right) \text { if } m>n+1,\end{cases}
$$


where $1 \leqq p \leqq n$. In particular, for $1 \leqq p \leqq n, H_{m-n+1}\left(\wedge^{p} f, E\right)=0$ if and only if Supp Coker $f \cap A s(E)=\varnothing$.

Proof. The case where $m=n$ or $m=n+1$ is very easy to verify, and thus we assume that $m>n+1$. Then the problem boils down to showing that

$$
\begin{aligned}
\operatorname{Ker}(d: & \left.\wedge^{q} R^{n *} \otimes \underset{v}{\ldots} \otimes R^{n *} \otimes \wedge^{m} R^{m} \otimes E \rightarrow \wedge R^{n *} \otimes \underset{v}{\ldots} \otimes \wedge R^{n *} \otimes \wedge R^{m} \otimes E\right) \\
& =\operatorname{Hom}\left(\Gamma_{q, v}(f), E\right)
\end{aligned}
$$

where

$d\left(\lambda_{0} \otimes \lambda_{1} \otimes \cdots \otimes \lambda_{v} \otimes \eta \otimes a\right)$

$=\sum_{i=0}^{v}(-1)^{i} \lambda_{0} \otimes \cdots \otimes \lambda_{i} \wedge \lambda_{i+1} \otimes \cdots \otimes \lambda_{v} \otimes \eta \otimes a+\lambda_{0} \otimes \cdots \otimes \lambda_{v-1} \otimes \omega_{\lambda v}(\eta) \otimes a$ with $\lambda_{0} \in \wedge^{q} R^{n *}, \lambda_{i} \in R^{n *}$ for $i=1, \cdots, v, \eta \in \wedge^{m} R^{m}$, and $a \in E$.

Let $x_{1}, \cdots, x_{n}$ and $y_{1}, \cdots, y_{m}$ be free bases of $R^{n}$ and $R^{m}$, respectively, and let $\xi_{1}, \cdots, \xi_{n}$ be the dual basis of $x_{i}, \cdots, x_{n}$. Then each element $\alpha$ in

$$
\wedge^{q} R^{n *} \otimes R^{n *} \otimes \underset{v}{\cdots} \otimes R^{n *} \otimes \bigwedge^{m} R^{m} \otimes E
$$

is uniquely written as

$$
\alpha=\sum \xi_{i_{1}} \wedge \cdots \wedge \xi_{i_{n}} \otimes \xi_{j_{1}} \otimes \cdots \otimes \xi_{j_{v}} \otimes \eta \otimes h\left(x_{i_{1}} \wedge \cdots \wedge x_{i_{q}}, x_{j_{1}}, \cdots, x_{j_{v}}\right)
$$

where $\eta=y_{1} \wedge \cdots \wedge y_{m}$ and $h\left(x_{i_{1}} \wedge \cdots \wedge x_{i_{q}}, x_{j_{1}}, \cdots, x_{j_{v}}\right)$ is in $E$. Then

$$
\begin{aligned}
& d(\alpha)=\sum \xi_{i_{1}} \wedge \cdots \wedge \xi_{i_{q}} \wedge \xi_{j_{1}} \otimes \xi_{j_{2}} \otimes \cdots \otimes \xi_{j_{v}} \otimes \eta \otimes h\left(x_{i_{1}} \wedge \cdots \wedge x_{i_{-}}, x_{j_{1}}, \cdots, x_{j_{v}}\right) \\
& +\sum_{k=1}^{v-1} \Sigma(-1)^{k} \xi_{i_{1}} \wedge \cdots \wedge \xi_{i_{q}} \otimes \xi_{j_{1}} \otimes \cdots \otimes \xi_{j_{k}} \wedge \xi_{j_{k+1}} \otimes \cdots \otimes \xi_{j_{v}} \otimes \eta \\
& \otimes h\left(x_{i_{1}} \wedge \cdots \wedge x_{i_{q}}, x_{j_{1}}, \cdots, x_{j_{v}}\right) \\
& +(-1)^{\nu} \sum \xi_{i_{1}} \wedge \cdots \wedge \xi_{i_{q}} \otimes \xi_{j_{1}} \otimes \cdots \otimes \xi^{j_{\nu}-1} \otimes \omega_{\xi_{j_{v}}}(\eta) \\
& \otimes h\left(x_{i_{1}} \wedge \cdots \wedge x_{i_{q}}, x_{j_{1}}, \cdots, x_{j_{v}}\right) \text {. }
\end{aligned}
$$

Therefore $d(\alpha)=0$ if and only if $h$ in $\operatorname{Hom}\left(\bigwedge^{q} R^{n} \otimes R^{n} \otimes \cdots \otimes R^{n}, E\right)$ satisfies the following three conditions:

(1) for each $i_{1}<\cdots<i_{q+1}, \Sigma_{k}(-1)^{k} h\left(x_{i_{1}} \wedge \cdots \wedge \hat{x}_{i_{k}} \wedge \cdots \wedge x_{i_{q+1}}, x_{i_{k}}, x_{j_{2}}, \cdots, x_{j_{v}}\right)=0$,

(2) $h\left(x_{i_{1}} \wedge \cdots \wedge x_{i_{q}}, x_{j_{1}}, \cdots, x_{j_{l}}, x_{j_{l+1}}, \cdots, x_{j_{v}}\right)=$

$$
h\left(x_{i_{1}} \wedge \cdots \wedge x_{i_{q}}, x_{j_{1}}, \cdots, x_{j_{t+1}}, x_{j_{1}}, \cdots, x_{j .}\right),
$$

(3) $\sum_{j_{v}} \omega_{\xi_{j_{v}}}(\eta) \otimes h\left(x_{i_{1}} \wedge \cdots \wedge x_{i_{q}}, x_{j l}, \cdots, x_{j_{v}}\right)=0$, i.e., $\sum_{j} \omega_{\xi_{j}}\left(y_{i}\right) h\left(x_{i_{1}} \wedge \cdots \wedge x_{i_{\curvearrowleft}}, x_{j_{1}}, \cdots, x_{j_{\ldots-1}}, x_{j_{v}}\right)=0$. 
Condition (2) says that $h$ is in $\operatorname{Hom}\left(\wedge^{q} R^{n} \otimes S_{v}\left(R^{n}\right), E\right)$. Then the conditions (1) and (3) mean that $h=0$ on $\operatorname{Im}\left(k_{q, v}: \wedge^{q+1} R^{n} \otimes S_{v-1}\left(R^{n}\right) \rightarrow \wedge^{q} R^{n} \otimes S_{v}\left(R^{n}\right)\right)$ and also on $\wedge^{q} R^{n} \otimes f\left(R^{m}\right) \cdot S_{v-1}\left(R^{n}\right)$. Therefore conditions (1), (2), and (3) simply mean that $h$ is in $\operatorname{Hom}\left(\wedge^{q} R^{n} \otimes S_{v}(\operatorname{Coker} f), E\right)$ and $h=0$ on

$$
\operatorname{Im}\left(k_{q, v}: \wedge^{q+1} R^{n} \otimes S_{v-1}(\text { Coker } f) \rightarrow \wedge^{q} R^{n} \otimes S_{v}(\text { Coker } f)\right),
$$

in other words, $h$ is in $\operatorname{Hom}\left(\Gamma_{q, v}(f), E\right)$. This proves our first assertion. The second statement then follows immediately from 2.2, namely that $\operatorname{Supp} \Gamma_{n+1-p, m-n-1}(f)=\operatorname{Supp} \operatorname{Coker} \wedge^{p} f=\operatorname{Supp}$ Coker $f$, for all $p$ such that $1 \leqq p \leqq n$.

Given a map $f: R^{m} \rightarrow R^{n}$, we denote by $I(f)$ the ideal generated by all the $n \times n$ minors of $f$, i.e., $I(f)=\operatorname{Ann}\left(\right.$ Coker $\left.\wedge^{n} f\right)$. We observe that Supp Coker $f=$ set of all prime ideals in $R$ containing $I(f)$. Therefore to say Supp Coker $f \neq \varnothing$ is the same thing as to say that $I(f) \neq R$ or that $f$ is not onto.

THEOREM 2.4. Given a map $f: R^{m} \rightarrow R^{n}(m \geqq n)$ and an $R$-module $E$ such that $E / I(f) E \neq 0$, we have for each $p$ with $1 \leqq p \leqq n$, the following statements:

(1) depth $(I(f) ; E)=$ the smallest integer $q$ for which $H^{q}\left(\wedge^{p} f, E\right) \neq 0$, and furthermore $H^{d}\left(\wedge^{p} f, E\right)=\operatorname{Ext}{ }^{d}\left(\right.$ Coker $\left.\wedge^{p} f, E\right)$ where $d=$ depth $(I(f) ; E)$.

(2) $m-n+1-\operatorname{depth}(I(f) ; E)=$ the largest integer $q$ for which $H_{q}\left(\wedge^{p} f, E\right) \neq 0$. Furthermore

$$
H_{m-n+1-d}\left(\bigwedge^{p} f, E\right)= \begin{cases}\operatorname{Ext}^{d}\left(\text { Coker } \wedge^{m-p} f, E\right) & \text { if } m=n \text { or } n+1, \\ \operatorname{Ext}^{d}\left(\Gamma_{n+1-p, m-n-1}(f), E\right) \text { if } m>n+1 .\end{cases}
$$

Proof. (1) The first statement follows immediately from 2.1 since for each $p$ such that $1 \leqq p \leqq n$, the functors $H^{i}\left(\wedge^{p} f, E\right)(i \geqq 0)$ satisfy the conditions of 2.1 (as can be seen by applying 1.5 ), with respect to the ideal $I(f)$. Furthermore, $H^{0}\left(\wedge^{p} f, E\right)=\operatorname{Hom}\left(\operatorname{Coker} \wedge^{p} f, E\right)$ and hence the second part of statement (1) also follows from 2.1 .

(2) Set $T^{i}(E)=H_{m-n+1-i}\left(\wedge^{p} f, E\right)$ where $1 \leqq p \leqq n$. Then it follows from 1.5 and 2.3 that $T^{i}(i \geqq 0)$ satisfy the conditions of 2.1 with respect to the ideal $I(f)$. Therefore depth $(I(f) ; E)=$ the smallest integer $q$ for which

$$
0 \neq T^{q}(E)=H_{m-n+1-q}\left(\bigwedge^{p} f, E\right),
$$

and hence $m-n+1-d$ is the largest integer $q$ for which $H_{q}\left(\bigwedge^{p} f, E\right) \neq 0$. The second statement follows from 2.1 and 2.3.

Corollary 2.5. $\sup _{E}$ depth $(I(f) ; E) \leqq m-n+1$ where E runs through all $R$-modules such that $E / I(f) E \neq 0$.

REMARK. The fact that depth $(I(f) ; R) \leqq m-n+1$ was proven by J. Eagon [6], the case when $R$ is a regular local ring being previously proven by Cohen [5]. 
However, we shall prove a stronger result in the next section, namely that $\sup _{\mathfrak{p}} \operatorname{dim} R_{\mathfrak{p}} \leqq m-n+1$ where $\mathfrak{p}$ runs through all minimal primes containing $I(f)$ (see also [6]).

COROLlaRY 2.6. Given a map $f: R^{m} \rightarrow R^{n}(m \geqq n)$ and an $R$-module $E$ with $E / I(f) E \neq 0$, the following statements are equivalent:

(1) for some $p, 1 \leqq p \leqq n, H_{q}\left(\wedge^{p} f, E\right)=0$ for all $q \neq 0$,

(2) for some $p, 1 \leqq p \leqq n, H^{q}\left(\wedge^{p} f, E\right)=0$ for all $q \neq m-n+1$,

(3) for all $p, 1 \leqq p \leqq n, H_{q}\left(\wedge^{p} f, E\right)=0$ for all $q \neq 0$ and $H^{q}\left(\wedge^{p} f, E\right)=0$ for all $q \neq m-n+1$,

(4) $\operatorname{depth}(I(f) ; E)=m-n+1$.

In particular, if Coker $f \neq 0, K\left(\wedge^{p} f\right)$ is an acyclic free resolution of Coker $\wedge^{p} f$ for some $p, 1 \leqq p \leqq n$ (or for all $p, 1 \leqq p \leqq n$ ) if and only if $\operatorname{depth}(I(f) ; R)$ $=m-n+1$.

COROLlaRY 2.7. Given a mapf: $R^{m} \rightarrow R^{n}$ with Coker $f \neq 0$, if $\operatorname{depth}(I(f) ; R)$ $=m-n+1$, then hd Coker $\wedge^{p} f=m-n+1$ for all $p, 1 \leqq p \leqq n$.

Proof. If depth $(I(f) ; R)=m-n+1$, then $K\left(\wedge^{p} f\right)$ is a free resolution of Coker $\wedge^{p} f$ and hence $h d$ Coker $\wedge^{p} f \leqq m-n+1 \quad(1 \leqq p \leqq n)$. But $\operatorname{depth}(I(f) ; R)=m-n+1$ implies that $H^{m-n+1}\left(\wedge^{p} f, E\right) \neq 0$, and hence $m-n+1$ $\leqq h d$ Coker $\wedge^{p} f$.

Another consequence of 2.4 is the generalized Cohen-Macaulay unmixedness theorem due to Eagon [6]. Indeed, we obtain the unmixedness theorem of Coker $\wedge^{\prime \prime} f$ for all $p, 1 \leqq p \leqq n$. An $R$-module $M$ is called Cohen-Macaulay if $\operatorname{depth} M=\operatorname{dim} M$. A ring $R$ which is Cohen-Macaulay as an $R$-module is called a Cohen-Macaulay ring [2]. We observe that if $M$ is a Cohen-Macaulay module, then $M$ is equidimensional, i.e., $\operatorname{dim} R / \mathfrak{p}$ is a constant (and equal to $\operatorname{dim} M$ ) for all $\mathfrak{p}$ in $A s(M)$. We also observe that, over a Cohen-Macaulay ring, the equidimensionality is the same as the unmixedness since $\operatorname{dim} R_{\mathfrak{p}}+\operatorname{dim} R / \mathfrak{p}=\operatorname{dim} R$ for all prime ideals $\mathfrak{p}[2]$.

Lemma 2.8. If $E$ is a Cohen-Macaulay R-module, then $\operatorname{Ext}_{R}^{d}(M, E)$ is equidimensional for any module $M$ such that $M \otimes E \neq 0$, where $d$ $=\operatorname{depth}(\operatorname{Ann}(M) ; E)$.

Proof. From the fact (which can be easily seen by localization) that $A s \operatorname{Hom}(M, N)=\operatorname{Supp} M \cap A s N$ it is clear that if $N$ is equidimensional, then so is $\operatorname{Hom}(M, N)$ for any $M$. Now let $I=\operatorname{Ann}(M)$. Then $M \otimes E \neq 0$ implies that $E / I E \neq 0$ and hence $d=\operatorname{depth}(I ; E)<\infty$. By 2.1 ,

$$
\operatorname{Ext}_{R}^{d}(M, E)=\operatorname{Hom}\left(M, E /\left(\alpha_{1}, \cdots, \alpha_{d}\right) E\right)
$$

where $\alpha_{1}, \cdots, \alpha_{d}$ is some proper $E$-sequence contained in $I$. If $E$ is Cohen-Macaulay, 
then so is $E /\left(\alpha_{1}, \cdots, \alpha_{d}\right) E$. Therefore $E /\left(\alpha_{1}, \cdots, \alpha_{d}\right) E$ is equidimensional and so is $\operatorname{Ext}^{d}(M, E)=\operatorname{Hom}\left(M, E /\left(\alpha_{1}, \cdots, \alpha_{a}\right) E\right)$.

THEOREM 2.9. L.et $f: R^{m} \rightarrow R^{n}(m \geqq n)$ be a map such that depth $(I(f) ; R)$ $=m-n+1$. Then, if $R$ is a Cohen-Macaulay ring, Coker $\wedge^{p} f$ is unmixed for all $p, 1 \leqq p \leqq n$.

Proof. If depth $(I(f) ; R)=m-n+1$, then $K\left(\wedge^{p} f\right)$ is a free resolution of Coker $\wedge^{p} f$, and $K\left(\wedge^{p} f\right)^{*}=\operatorname{Hom}\left(K\left(\wedge^{p} f\right), R\right)$ is a free resolution of $H^{m-n+1}\left(\wedge^{p} f, R\right)$ by 2.6. Therefore,

$$
\operatorname{Ext}^{i}\left(H^{m-n+1}\left(\wedge^{p} f, R\right), R\right)=\left\{\begin{array}{lr}
0 & \text { if } i<m-n+1, \\
\text { Coker } \wedge^{p} f \text { if } i=m-n+1 .
\end{array}\right.
$$

Consequently, depth $(J ; R)=m-n+1$ where $J=\operatorname{Ann}\left(H^{m-n+1}\left(\wedge^{p} f, R\right)\right)$ by 2.1. Thus by 2.8, Coker $\wedge^{p} f=\operatorname{Ext}^{m-n+1}\left(H^{m-n+1}\left(\wedge^{p} f, R\right), R\right)$ is equidimensional and hence unmixed if $R$ is a Cohen-Macaulay ring.

3. Hilbert characteristic function associated with a matrix. Before we go any further, let us fix our notation. If $A$ is a graded ring (or graded module), $A_{v}$ will denote the $v$ th homogeneous component of $A$. For each $R$-module $M$, $S(M)=\Sigma_{v \geq 0} S_{v}(M)$ will denote the symmetric algebra generated by $M$ over $R$. The natural identification $M=S(M)_{1}$ gives us the canonical $S(M)$-homomorphism $\tau_{M}: S(M) \otimes M \rightarrow S(M)$. In the case when $M=R^{m}$, we shall simply write $\tau^{m}$ instead of $\tau_{R^{m}}$, and thus $K\left(\tau^{m}\right)$ is the (usual) Koszul complex associated with the map $\tau^{m}: S\left(R^{m}\right)^{m} \rightarrow S\left(R^{m}\right)$.

Given a map $f: R^{m} \rightarrow R^{n}$, we get the algebra map $S(f): S\left(R^{m}\right) \rightarrow S\left(R^{n}\right)$ and, for each $R$-module $E, S\left(R^{n}\right) \otimes E$ becomes a module over $S\left(R^{m}\right)$, thus $H\left(\tau^{m}, S\left(R^{n}\right) \otimes E\right)$ may be considered. We observe that $H\left(\tau^{m}, S\left(R^{n}\right) \otimes E\right)$ is a graded module over the graded algebra $S\left(R^{n}\right)$.

Now suppose that $l($ Coker $f \otimes E)<\infty$ (where $l(*)$ means the length of the $R$-module $*)$. Then it follows from Supp Coker $f=\operatorname{Supp}$ Coker $S_{v}(f)(v>0)$ that $l\left(\right.$ Coker $\left.S_{v}(f) \otimes E\right)<\propto$ for all $v>0$. Therefore, we can define the function $P_{f}(v, E)=l\left(\right.$ Coker $\left.S_{v}(f) \otimes E\right)$ from the set of positive integers into itself. One of the main purposes of this section is to show that $P_{f}(v, E)$ is a polynomial function, and thereby to obtain a more general notion of multiplicity.

THEOREM 3.1. Let $f: R^{m} \rightarrow R^{n}$ be a map, and $E$ an $R$-module such that $l($ Coker $f \otimes E)<\infty$. Then $P_{f}(v, E)$ is a polynomial function for sufficiently large v. Furthermore, $\Delta^{m} P_{f}(v, E)=\Sigma_{q}(-1)^{m-q} l\left(H_{m-q}\left(\tau^{m}, S\left(R^{n}\right) \otimes E\right)_{v+q}\right)$ for all sufficiently large $v$, where $\Delta P(v)$ is defined to be $P(v+1)-P(v)$ and $\Delta^{i} P(v)$ $=\Delta\left(\Delta^{i-1} P(v)\right)$.

Proof. Consider the exact sequence of $S\left(R^{m}\right)$-modules:

$$
0 \rightarrow S\left(R^{m}\right) \cdot E \rightarrow S\left(R^{n}\right) \otimes E \rightarrow \operatorname{Coker} S(f) \otimes E \rightarrow 0
$$


where $S\left(R^{m}\right) E=\operatorname{Im}\left(S(f) \otimes E: S\left(R^{m}\right) \otimes E \rightarrow S\left(R^{n}\right) \otimes E\right)$. One may notice that Coker $S(f) \otimes E$ is in general nct finitely generated over $S\left(R^{m}\right)$. However, $S\left(R^{m}\right) E$ is finitely generated over $S\left(R^{m}\right)$ and hence $H_{*}\left(\tau^{m}, S\left(R^{m}\right) E\right)$ is a finitely generated $S\left(R^{m}\right)$-module annihilated by the ideal $\operatorname{Im} \tau^{m}$. Therefore, $H_{*}\left(\tau^{m}, S\left(R^{m}\right) E\right)$ is finitely generated over $S\left(R^{m}\right) / \operatorname{Im} \tau^{m}=R$ and hence $H_{*}\left(\tau^{m}, S\left(R^{m}\right) E\right)_{v}=0$ for all sufficiently large $v$. On the other hand, since the differentiation map on the Koszul complex $K\left(\tau^{m}\right)$ raises the homogeneous degree by one, we see that each homogeneous part of the chain complex $K\left(\tau^{m}\right) \otimes_{\left(S\left(R^{m}\right)\right)}$ Coker $S(f) \otimes E$ stable under the differentiation is of the form:

$$
\begin{aligned}
\wedge^{m} R^{m} \otimes \operatorname{Coker} S_{v}(f) \otimes E & \rightarrow \wedge^{m-1} R^{m} \otimes \operatorname{Coker} S_{v+1}(f) \otimes E \rightarrow \cdots \\
\cdots & \rightarrow \wedge^{m-\epsilon_{1}} R^{m} \otimes \operatorname{Coker} S_{v+q}(f) \otimes E \rightarrow \cdots \\
\cdots & \rightarrow \wedge^{0} R^{m} \otimes \operatorname{Coker} S_{v+m}(f) \otimes E .
\end{aligned}
$$

Since the Euler-Poincaré characteristic is preserved by passage to homology, we get

$$
\begin{aligned}
\Delta^{m} P_{f}(v, E) & =\sum_{q=0}^{m}(-1)^{m-q}\left(\begin{array}{c}
m \\
q
\end{array}\right) l\left(\operatorname{Coker} S_{v+q}(f) \otimes E\right) \\
& =\sum_{q=0}^{m}(-1)^{m-q} l\left(H_{m-q}\left(\tau^{m}, \text { Coker } S(f) \otimes E\right)_{v+q}\right) \\
& =\sum_{q=0}^{m}(-1)^{m-q} l\left(H_{m-\imath}\left(\tau^{m}, S\left(R^{n}\right) \otimes E\right)_{v+q}\right) .
\end{aligned}
$$

This establishes our second assertion.

Now consider the double complex $X=K\left(\tau^{n}\right) \otimes_{S_{\left(R^{n}\right)}}\left(K\left(\tau^{m}\right) \otimes_{S\left(R^{m}\right)} S\left(R^{n}\right) \otimes E\right)$. Then $H(X)$ is a finitely generated $S\left(R^{n}\right)$-module annihilated by the ideal $\operatorname{Im} \tau^{n}$ and hence is a finitely generated module over $S\left(R^{n}\right) / \operatorname{Im} \tau^{n}=R$. Consequently $H(X)_{v}=0$ for all sufficiently large $v$. On the other hand, it is clear that the $E_{1}$ term of the spectral sequence of this double complex $X$ is simply given by $E_{1}^{p, q}=\wedge^{p} R^{n} \otimes H_{q}\left(\tau^{m}, S\left(R^{n}\right) \otimes E\right)$. Since the Euler-Poincaré characteristic is preserved throughout the spectral sequence, we obtain (using the fact that $H(X)_{v}=0$ for all sufficiently large $v$ ) that

$$
\begin{aligned}
0 & =\sum_{i}(-1)^{m+n-i} l\left(H_{m+n-i}(X)_{v+i}\right)=\sum_{p, q}(-1)^{m+n-p-q} l\left(\left(E_{1}^{n-p, m-q}\right)_{v+p+q}\right) \\
& =\sum_{p, q}(-1)^{m+n-p-q}\left(\begin{array}{l}
n \\
p
\end{array}\right) l\left(H_{m-q}\left(\tau^{m}, S\left(R^{n}\right) \otimes E\right)_{v+p+q}\right) \\
& =\sum_{p}(-1)^{n-p}\left(\begin{array}{l}
n \\
p
\end{array}\right)\left[\sum_{q}(-1)^{m-q} l\left(H_{m-q}\left(\tau^{m}, S\left(R^{n}\right) \otimes E\right)_{v+p+q}\right)\right] \\
& =\sum(-1)^{n-p}\left(\begin{array}{l}
n \\
p
\end{array}\right) \Delta^{m} P_{f}(v+p, E)=\Delta^{m+n} P_{f}(v, E)
\end{aligned}
$$


when $v$ is sufficiently large. This proves that $P_{f}(v, E)$ is a polynomial function for sufficiently large $v$.

We now consider the degree and the leading coefficient of this polynomial function $P_{f}(v, E)$.

For each polynomial function $\phi$, we set $u(\phi)=(\operatorname{deg} \phi)$ ! (the leading coefficient of $\phi$ ). Thus we observe that if $\phi(v)$ is a positive integer for all sufficiently large integers $v$, then $u(\phi)$ is a positive integer.

LEMMA 3.2. If $\phi_{1}, \phi_{2}$ are polynomial functions, then the function $\phi_{1} * \phi_{2}$ defined by $\phi_{1} * \phi_{2}(v)=\sum_{p+y=v} \phi_{1}(p) \phi_{2}(q)$ is also a polynomial function and we have that $\operatorname{deg} \phi_{1} * \phi_{2}=\operatorname{deg} \phi_{1}+\operatorname{deg} \phi_{2}+1$. We also have $u\left(\phi_{1} * \phi_{2}\right)=u\left(\phi_{1}\right) u\left(\phi_{2}\right)$.

Proof. For each integer $n \geqq 0$, we set

Then

$$
P_{n}(X)=\left(\begin{array}{c}
X+n \\
n
\end{array}\right)
$$

$$
P_{n_{1}} * P_{n_{2}}(v)=\sum_{p+q=v}\left(\begin{array}{c}
p+n_{1} \\
n_{1}
\end{array}\right)\left(\begin{array}{c}
q+n_{2} \\
n_{2}
\end{array}\right)=\left(\begin{array}{c}
v+n_{1}+n_{2}+1 \\
n_{1}+n_{2}+1
\end{array}\right)
$$

i.e., $P_{n_{1}} * P_{n_{2}}=P_{n_{1}+n_{2}+1}$. We prove our lemma by induction on $\operatorname{deg} \phi_{1}+\operatorname{deg} \phi_{2}$, the case $\operatorname{deg} \phi_{1}+\operatorname{deg} \phi_{2}=0$ being trivial.

We can always write $\phi_{i}=u\left(\phi_{i}\right) P_{d_{i}}+\phi_{i}^{\prime}$ where $\operatorname{deg} \phi_{i}^{\prime}<d_{i}=\operatorname{deg} \phi_{i}$. Then $\phi_{1} * \phi_{2}=u\left(\phi_{1}\right) u\left(\phi_{2}\right) P_{d_{1}+d_{2}+1}+\left[u\left(\phi_{1}\right) P_{d_{1}} * \phi_{2}^{\prime}+u\left(\phi_{2}\right) \phi_{1}^{\prime} * P_{d_{2}}+\phi_{1}^{\prime} * \phi_{2}^{\prime}\right]$ and inside the bracket is a polynomial function of degree less than $d_{1}+d_{2}+1$, by induction. Hence $\phi_{1} * \phi_{2}$ is a polynomial function of degree $d_{1}+d_{2}+1$ and $u\left(\phi_{1} * \phi_{2}\right)=u\left(\phi_{1}\right) u\left(\phi_{2}\right)$.

THEOREM 3.3. Let $f: R^{m} \rightarrow R^{n}$ be a map, and $E$ an $R$-module such that $l($ Coker $f \otimes E)<\infty$. Then $u\left(P_{f}(v, E)\right)$ and $\operatorname{deg} P_{f}(v, E)-n+1$ depend only on the $R$-modules $E$ and Coker $f$.

Proof. It suffices to show that if $R^{m_{i}} \stackrel{f_{i}}{\rightarrow} R^{n_{i}} \rightarrow M \rightarrow 0(i=1,2)$ are exact sequences, and $l(M \otimes E)<\infty$, then $P_{n_{2}-1} * P_{f_{1}}=P_{n_{1}-1} * P_{f_{2}}$ where

$$
P_{n}(v)=\left(\begin{array}{c}
v+n \\
n
\end{array}\right), \quad P_{f_{i}}(v)=P_{f_{i}}(v, E)
$$

Consider the exact sequences

$$
\begin{aligned}
& 0 \rightarrow f_{1}\left(R^{m_{1}}\right) \stackrel{g_{1}}{\rightarrow} R^{n_{1}} \stackrel{\pi_{1}}{\rightarrow} M \rightarrow 0 \\
& 0 \rightarrow f_{2}\left(R^{m_{2}}\right) \stackrel{g_{2}}{\rightarrow} R^{n_{2}} \stackrel{\pi_{2}}{\rightarrow} M \rightarrow 0
\end{aligned}
$$

where $g_{i}$ are inclusion maps. We observe that Coker $S_{v}\left(f_{i}\right)=\operatorname{Coker} S_{v}\left(g_{i}\right)$ for all $v$ since $\operatorname{Im} f_{i}=\operatorname{Im} g_{i}(i=1,2)$. If we set $K=\left\{\left(x_{1}, x_{2}\right) \in R^{n_{1}} \oplus R^{n_{2}} \mid \pi_{1}\left(x_{1}\right)=\pi_{2}\left(x_{2}\right)\right\}$, then we get the exact sequence 


$$
0 \rightarrow K \stackrel{g}{\rightarrow} R^{n_{1}} \oplus R^{n_{2}} \rightarrow M \rightarrow 0 \quad(g \text { is inclusion map) }
$$

and the commutative diagram

$$
\begin{aligned}
& f_{1}\left(R^{m_{1}}\right) \oplus R^{n_{2}} \stackrel{\approx}{\rightarrow} K \underset{\leftarrow}{\approx} R^{n_{1}} \oplus f_{2}\left(R^{m_{2}}\right) \\
& g_{1}+i d \downarrow \downarrow g \quad \downarrow i d+g_{2} \\
& R^{n_{1}} \oplus R^{n_{2}} \stackrel{\text { id }}{\rightarrow} R^{n_{1}} \oplus R^{n_{2}} \rightarrow R^{n_{1}} \oplus R^{n_{2}} .
\end{aligned}
$$

Therefore we have

Coker $S_{v}(g) \otimes E=\operatorname{Coker} S_{v}\left(g_{1}+i d\right) \otimes E=\sum_{p+q=v} S_{p}\left(R^{n n_{2}}\right) \otimes \operatorname{Coker} S_{q}\left(g_{1}\right) \otimes E$

$$
=\sum_{p+q=v} S_{p}\left(R^{n_{2}}\right) \otimes \text { Coker } S_{q}\left(f_{1}\right) \otimes E
$$

Coker $S_{v}(g) \otimes E=$ Coker $S_{v}\left(i d+g_{2}\right) \otimes E=\sum_{p+y=v} S_{p}\left(R^{n_{1}}\right) \otimes \operatorname{Coker} S_{q}\left(g_{2}\right) \otimes E$

Hence

$$
=\sum_{p+q=v} S_{p}\left(R^{n_{1}}\right) \otimes \text { Coker } S_{q}\left(f_{2}\right) \otimes E
$$

$$
\sum_{p+q=v}\left(\begin{array}{c}
p+n_{1}-1 \\
n_{1}-1
\end{array}\right) P_{f_{2}}(q, E)=\sum_{p+q=v}\left(\begin{array}{c}
p+n_{2}-1 \\
n_{2}-1
\end{array}\right) P_{f_{1}}(q, E) \text { for all } v
$$

i.e., $P_{n_{1}-1} * P_{f_{2}}=P_{n_{2}-1} * P_{f_{1}}$.

THEOREM 3.4. Let $R$ be a local ring, $f: R^{m} \rightarrow R^{n}$ a map, and $E$ an $R$-module such that $l($ Coker $f \otimes E)<\infty$. Then $\operatorname{deg} P_{f}(v, E)=n-1+\operatorname{dim} E$.

Proof. Since $\operatorname{deg} P_{f}(v, E)-n+1$ depends only on Coker $f$ (for a fixed $E$ ), we may assume that $\operatorname{Im} f \subset \mathfrak{m} R^{n}$, where $m$ is the maximal ideal of $R$. Let $g_{1}: R^{m_{1}} \rightarrow R^{n}$ and $g_{2}: R^{m_{2}} \rightarrow R^{n}$ be maps such that $\operatorname{Im} g_{i}=\mathfrak{q}_{i} R^{n}$ where $\mathfrak{q}_{1}=\mathrm{m}$, $\mathfrak{q}_{2}=I(f)$. Then we have $\operatorname{Im} g_{2} \subset \operatorname{Im} f \subset \operatorname{Im} g_{1}$ and hence $l\left(\right.$ Coker $\left.S_{v}\left(g_{2}\right) \otimes E\right)$ $\geqq l\left(\right.$ Coker $\left.S_{v}(f) \otimes E\right) \geqq l\left(\right.$ Coker $\left.S_{v}\left(g_{1}\right) \otimes E\right)$, i.e., $P_{g_{2}}(v, E) \geqq P_{f}(v, E) \geqq P_{g_{1}}(v, E)$ for all $v$. Thus $\operatorname{deg} P_{g_{2}} \geqq \operatorname{deg} P_{f} \geqq \operatorname{deg} P_{g_{1}}$. However, Coker $S_{v}\left(g_{i}\right)=S_{v}\left(R^{n}\right) / q_{i}^{v} S_{v}\left(R^{n}\right)$ and therefore

$$
P_{g_{i}}(v, E)=l\left(\text { Coker } S_{v}\left(g_{i}\right) \otimes E\right)=l\left(S_{v}\left(R^{n}\right) \otimes E / \mathfrak{q}_{i}^{v} E\right)=\left(\begin{array}{c}
v+n-1 \\
n-1
\end{array}\right) l\left(E / \mathfrak{q}_{i}^{v} E\right) .
$$

Therefore $\operatorname{deg} P_{g_{i}}(v, E)=n-1+\operatorname{dim} E$ for $i=1,2$ and consequently $\operatorname{deg} P_{f}(v, E)$ $=n-1+\operatorname{dim} E$.

The following theorem is a generalization of the Krull principal ideal theorem.

THEOREM 3.5. Let $R$ be an arbitrary noetherian ring. Then for each map $f: R^{m} \rightarrow R^{n}(m \geqq n)$, we have $\operatorname{dim} R_{\mathfrak{p}} \leqq m-n+1$ for all minimal primes $\mathfrak{p}$ in Supp Coker $f$. In particular, $m-n+1 \geqq h t$ Coker $f\left({ }^{4}\right)$.

(4) $h t M$ is here used to denote what we have previously called rank $M$, i.e., $\inf _{\mathfrak{p}} \operatorname{dim} R_{\mathfrak{p}}$ where $\mathfrak{p}$ runs through the primes in Supp $M$. 
Proof. Localizing at each minimal prime $\mathfrak{p}$ in $\operatorname{Supp}$ Coker $f$, it suffices to show that $\operatorname{dim} R \leqq m-n+1$ if $l(\operatorname{Coker} f)<\infty$. Furthermore we may assume that $R$ is an integral domain, for otherwise we may replace $R$ by $R / \mathfrak{p}$ where $\mathfrak{p}$ is a prime ideal such that $\operatorname{dim} R / \mathfrak{p}=\operatorname{dim} R$.

If $\operatorname{dim} R=0$, there is nothing to prove, so we may assume $\operatorname{dim} R>0$. $R$ being an integral domain of positive dimension, we know there is a nonzero element $\alpha$ in the maximal ideal of $R$. Then the exact sequence $0 \rightarrow R \stackrel{\alpha}{\rightarrow} R \rightarrow R / \alpha \rightarrow 0$ gives the exact sequence $0 \rightarrow S\left(R^{n}\right) \stackrel{\alpha}{\rightarrow} S\left(R^{\prime \prime}\right) \rightarrow S\left(R^{\prime \prime}\right) \otimes R / \alpha \rightarrow 0$, and it follows from the exact sequence $\cdots \rightarrow H_{p}\left(\tau^{m}, S\left(R^{n}\right)\right) \rightarrow H_{p}\left(\tau^{m}, S\left(R^{n}\right) \otimes R / \alpha\right) \rightarrow H_{p-1}\left(\tau^{m}, S\left(R^{n}\right)\right) \rightarrow \cdots$ that $\Sigma_{p}(-1)^{m-p} l\left(H_{m-p}\left(\tau^{m}, S\left(R^{n}\right) \otimes R / \alpha\right)_{n+p}\right)=0$ for all $v$. This means, by 3.1 that $\Delta^{m} P_{f}(v, R / \alpha)=0$, and hence $m>\operatorname{deg} P_{f}(v, R / \alpha)$. But by 3.4 , $\operatorname{deg} P_{f}(v, R / \alpha)=n-1+\operatorname{dim} R / \alpha$, and $\operatorname{dim} R / \alpha=\operatorname{dim} R-1$. Hence $m-1 \geqq n-2$ $+\operatorname{dim} R$ or $m-n+1 \geqq \operatorname{dim} R$.

COROLlaRY 3.6. Let $R$ be a local ring, $f: R^{m} \rightarrow R^{n}(m \geqq n)$ a map, and $E$ an $R$-module such that $l($ Coker $f \otimes E)<\infty$. Then $m-n+1 \geqq \operatorname{dim} E$ and hence $m \geqq \operatorname{deg} P_{f}(v, E)$.

Proof. Let $\bar{R}=R /$ Ann $(E)$ and $\bar{f}=f \otimes \bar{R}: \bar{R}^{m} \rightarrow \bar{R}^{n}$. Then $l($ Coker $f)<\infty$ and hence $m-n+1 \geqq \operatorname{dim} \bar{R}=\operatorname{dim} E$. Thus $m \geqq n-1+\operatorname{dim} E=\operatorname{deg} P_{f}(v, E)$.

For each module $M$ over a local ring $R$, define $n_{i}(M)=\left[\operatorname{Tor}_{i}(M, R / \mathrm{m}): R / \mathrm{m}\right]$, where $\mathrm{m}$ is the maximal ideal of $R$.

COROLlaRY 3.7. Let $R$ be a local ring, and $M$ a torsion module over $R$. Then $n_{1}(M)-n_{0}(M)+1 \geqq h t M$, and the equality holds if and only if there exists an exact sequence $R^{m} \rightarrow R^{n} \rightarrow M \rightarrow 0$ such that $m-n+1=h t M$. Moreover, if ht $M>1$ and the equality holds, then $M$ is indecomposable.

Proof. First observe that if $R^{m} \rightarrow R^{n} \rightarrow M \rightarrow 0$ is exact, then $m \geqq n$ since $M$ is a torsion module. Let $0 \rightarrow K_{1} \rightarrow F \rightarrow M \rightarrow 0$ be an exact sequence with $F$ a free $R$-module, and $K_{1} \subset \mathrm{m} F$. Then $\operatorname{Tor}_{1}(M, R / \mathrm{m})=K_{1} \otimes R / \mathrm{m}$. Now for any exact sequence $R^{m} \stackrel{f}{\rightarrow} R^{n} \rightarrow M \rightarrow 0$, we get $K_{1} \oplus R^{n} \approx F \oplus f\left(R^{m}\right)$ and thus $n_{1}(M)+n=n_{0}(M)+\left[f\left(R^{m}\right) \otimes R / m: R / m\right] \leqq n_{0}(M)+m$. Therefore $m-n+1$ $\geqq n_{1}(M)-n_{0}(M)+1 \geqq h t M$ (by 3.5). If for some exact sequence, $m-n+1=h t M$, then also $n_{1}(M)-n_{0}(M)+1=h t M$. Since one may always choose $m=n_{1}(M)$ and $n=n_{0}(M)$, it is clear that the converse also holds.

Now assume that $n_{1}(M)-n_{0}(M)+1=h t M$, and that $M=M_{1} \oplus M_{2}$. Then $h t M=n_{1}\left(M_{1}\right)-n_{0}\left(M_{1}\right)+1+n_{1}\left(M_{2}\right)-n_{0}\left(M_{2}\right)+1-1 \geqq h t M_{1}+h t M_{2}-1 \geqq 2 h t M-$ 1 or $h t M \leqq 1$. Thus if $h t M>1, M$ is indecomposable if $n_{1}(M)-n_{0}(M)+1=h t M$.

Our theorems naturally lead us to the following definitions.

Given a module $M$ of finite length over a local ring $R$, choose an exact sequence $R^{m} \stackrel{f}{\rightarrow} R^{n} \rightarrow M \rightarrow 0$. Then for each $R$-module $E$, the product $(\operatorname{dim} R+n-1)$ ! (the coefficient of the term of degree $n-1+\operatorname{dim} R$ in the polynomial $P_{f}(v, E)$ ) is a non-negative integer which depends only on $M$ and $E$. We call it the multi- 
plicity of $M$ with respect to $E$ and denote it by $e_{E}(M)$. In the case when $M=R$, we simply write $e(M)$. Thus when $M=R / \mathfrak{q}$, our notation $e(R / \mathfrak{q})$ means the multiplicity of $\mathfrak{q}$ in the usual sense.

In view of 3.6 , we call a map $f: R^{m} \rightarrow R^{n}$ a parameter matrix for $E$ if $l($ Coker $f \otimes E)<\infty$ and $m-n+1=\operatorname{dim} E$. When $n=1$, a parameter matrix for $E$ is nothing but a system of parameters for $E$. Our construction of a parameter matrix below shows that for each $E$, and each $n>0$, there exists a parameter matrix $f: R^{m} \rightarrow R^{n}$ for $E$.

This next proposition is an immediate consequence of our definitions and the theorems already established above.

PROPOSITION 3.8. Let $f: R^{m} \rightarrow R^{n}$ be a parameter matrix for $R$, and let $M=$ Coker $f$. Then

(1) $e_{E}(M)=\Sigma_{p}(-1)^{m-p} l\left(H_{m-p}\left(\tau^{m}, S\left(R^{n}\right) \otimes E\right)_{v+p}\right)$ where $v$ is sufficiently large.

(2) $e_{E}(M) \geqq 0$ and $e_{E}(M)=0$ if and only if $\operatorname{dim} E<\operatorname{dim} R$.

(3) If $0 \rightarrow E^{\prime} \rightarrow E \rightarrow E^{\prime \prime} \rightarrow 0$ is an exact sequence, then $e_{E}(M)=e_{E^{\prime}}(M)+e_{E^{\prime \prime}}(M)$.

We shall conclude this section with a fairly general construction of parameter matrices.

Let $\phi=\left\{a_{1}, \cdots, a_{d}\right\}$ be any sequence of $d$ elements in a commutative ring $R$, and let $n$ be any positive integer. From $\phi$, we construct an $(n+d-1) \times n$-matrix which we will denote by $\phi^{(n)}$. $\phi^{(n)}$ is defined as follows: $\phi^{(n)}(i, j)=a_{i-j}$ where $1 \leqq i \leqq n+d-1,0 \leqq j \leqq n-1$, and

$$
a_{i-j}= \begin{cases}0 & \text { if } i-j \leqq 0 \text { or if } i-j>d, \\ a_{i-j} & \text { if } 1 \leqq i-j \leqq d .\end{cases}
$$

Put a little more picturesquely, the matrix $\phi^{(n)}$ looks like this:

$$
\left[\begin{array}{ccccl}
a_{1} & 0 & 0 & \cdots & 0 \\
a_{2} & a_{1} & 0 & \cdots & 0 \\
\cdot & \cdot & \cdot & & \cdot \\
\cdot & \cdot & \cdot & & \cdot \\
\cdot & \cdot & \cdot & & \cdot \\
a_{d} & a_{d-1} & a_{d-2} & \cdots & a_{d-n+1} \\
0 & a_{d} & a_{d-1} & \cdots & a_{d-n+2} \\
0 & 0 & a_{d} & \cdots & a_{d-n+3} \\
\cdot & \cdot & \cdot & & \cdot \\
\cdot & \cdot & \cdot & & \cdot \\
\cdot & \cdot & \cdot & & \cdot \\
0 & 0 & 0 & \cdots & a_{d}
\end{array}\right]
$$


Choosing canonical bases for $R^{d+n-1}$ and for $R^{n}$, the matrix $\phi^{(n)}$ defines a map (which we will continue to call $\phi^{(n)}$ ) from $R^{d+n-1}$ to $R^{n}$. Corresponding to this map, we have the ideal $I\left(\phi^{(n)}\right)=$ Ann Coker $\wedge^{n} \phi^{(n)}$. The reason for considering the map $\phi^{(n)}$ becomes clear when we state the following lemma (without proof).

LEMMA 3.9. Let $\phi=\left\{a_{1}, \cdots, a_{d}\right\}$ be a sequence of elements of $R$, and $\phi^{(n)}$ the corresponding map, $\phi^{(n)}: R^{d+n-1} \rightarrow R^{n}$. Then $I\left(\phi^{(n)}\right)=J^{n}$ where $J$ is the ideal generated by $a_{1}, \cdots, a_{\downarrow}$. Thus, if $R$ is a local ring, $E$ an $R$-module of dimension $d$, and $a_{1}, \cdots, a_{d}$ a system of parameters for $E$, then $\phi^{(n)}$ is a parameter matrix for $E$ for every positive integer $n$.

In the next section we shall compute the multiplicity of some parameter matrices. Let us simply remark here that the existence of parameter matrices of arbitrary size proves (by 3.7) that if $R$ is a local ring of dimension greater than one, and if $n$ is any positive integer, then there exists an indecomposable module $M$ of finite length which is minimally generated over $R$ by $n$ elements.

4. Euler-Poincaré characteristic and multiplicity. The main purpose of this section is to establish the fact that if $f: R^{m} \rightarrow R^{n}$ is a map, and $E$ an $R$-module such that $l($ Coker $f \otimes E)<\infty$, then the Euler-Foincaré characteristic of $H_{*}\left(\wedge^{p} f, E\right)$ is equal to

$$
\left(\begin{array}{l}
n-1 \\
n-p
\end{array}\right) \Delta^{m} P_{f}(v, E)
$$

We obtain this result from a double complex, which will also be used in a subsequent paper.

Let us begin then with the construction of the double complex. Consider two maps $A \stackrel{f}{\rightarrow} B \stackrel{g}{\rightarrow} C$ of $R$-modules. Then $f: A \rightarrow B$ induces a map on bilinear forms $\left(\gamma(g f), A, C^{*}\right) \rightarrow\left(\gamma(g), B, C^{*}\right)$ and hence the chain map:

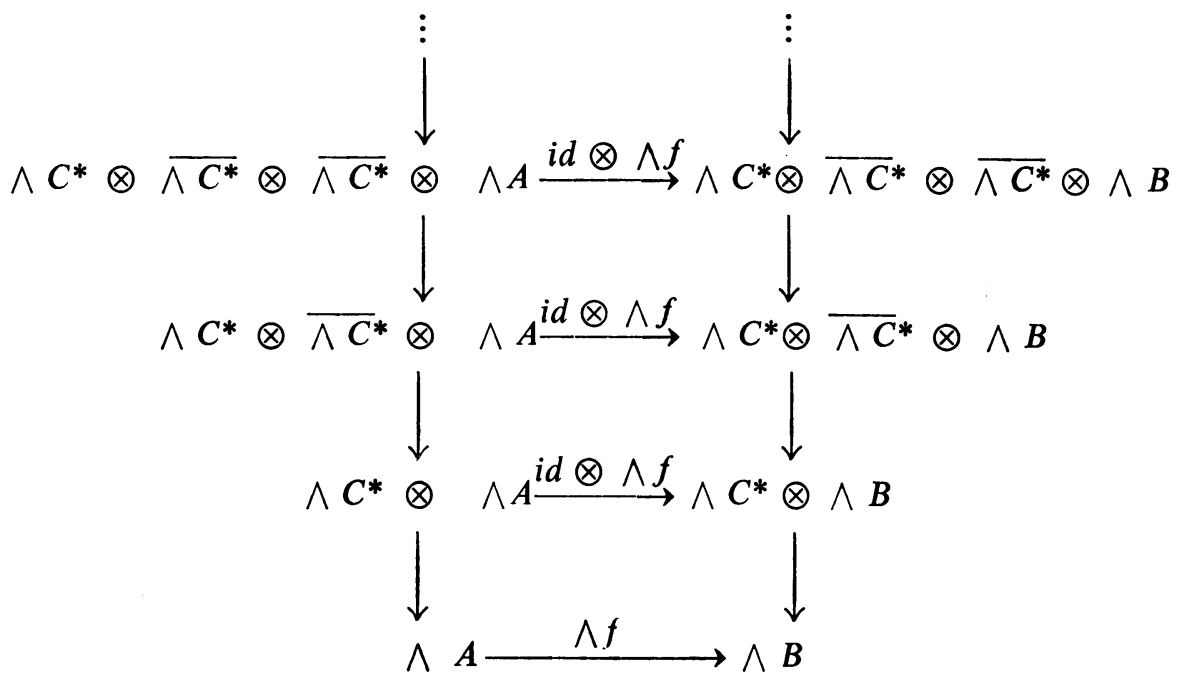


where $\overline{\wedge C}^{*}=\Sigma_{p>0} \wedge^{p} C^{*}$ and the vertical maps are defined, as in $\S 1$, by

$$
\begin{aligned}
d^{\prime}\left(\lambda_{0} \otimes \cdots \otimes \lambda_{p} \otimes \alpha\right)= & \sum_{i=0}^{p-1}(-1)^{i} \lambda_{0} \otimes \cdots \otimes \lambda_{i} \wedge \lambda_{i+1} \otimes \cdots \otimes \lambda_{p} \otimes \alpha \\
& +(-1)^{p} \lambda_{0} \otimes \cdots \otimes \lambda_{p-1} \otimes \omega_{\lambda_{p}}(\alpha) .
\end{aligned}
$$

In addition to this chain map between $\mathfrak{C}(\gamma(g f), \wedge A)$ and $\mathfrak{C}(\gamma(g), \wedge B)$, we also have the complex $\mathfrak{C}(\gamma(f), \wedge A)$. The double complex that we will ultimately construct will involve all of these complexes, so let us introduce scme more notation to simplify our writing.

For each $p \geqq 0$, let us define:

$$
\begin{aligned}
& L_{p}^{\prime}=\overline{\wedge C}^{*} \otimes \overline{\wedge C}^{*} \otimes \cdots \otimes \overline{\wedge C}^{*} \quad p \text { copies, } \\
& L_{p}=\wedge C^{*} \otimes L_{p}^{\prime},
\end{aligned}
$$

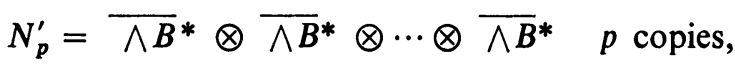

$$
\begin{aligned}
& N_{p}=\wedge B^{*} \otimes N_{p}^{\prime}
\end{aligned}
$$

In terms of this notation, let us observe that $\mathbb{C}_{q+1}(\gamma(f), \wedge A)=N_{q} \otimes \wedge A$, and that $\mathfrak{D}_{p+1}\left(g^{*}, \wedge B^{*}\right)=L_{p} \otimes \wedge B^{*}$, where $g^{*}: C^{*} \rightarrow B^{*}$ is the transpose of $g: B \rightarrow C$.

Consider now the diagram:
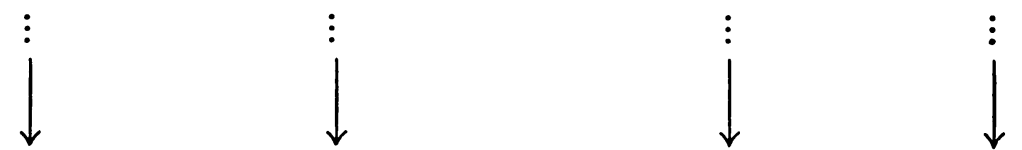

$\cdots \rightarrow \quad L_{p} \otimes N_{q} \otimes \wedge A \rightarrow \quad L_{p} \otimes N_{q-1} \otimes \wedge A \rightarrow \cdots \rightarrow \quad L_{p} \otimes N_{0} \otimes \wedge A \rightarrow L_{p} \otimes \wedge A$

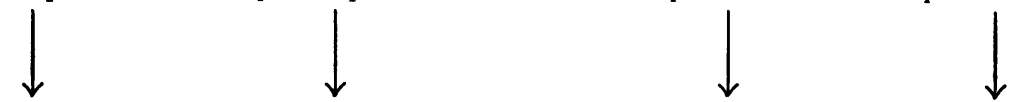

$\cdots \rightarrow L_{p-1} \otimes N_{q} \otimes \wedge A \rightarrow L_{p-1} \otimes N_{q-1} \otimes \wedge A \rightarrow \cdots \rightarrow L_{p-1} \otimes N_{0} \otimes \wedge A \rightarrow L_{p-1} \otimes \wedge A$

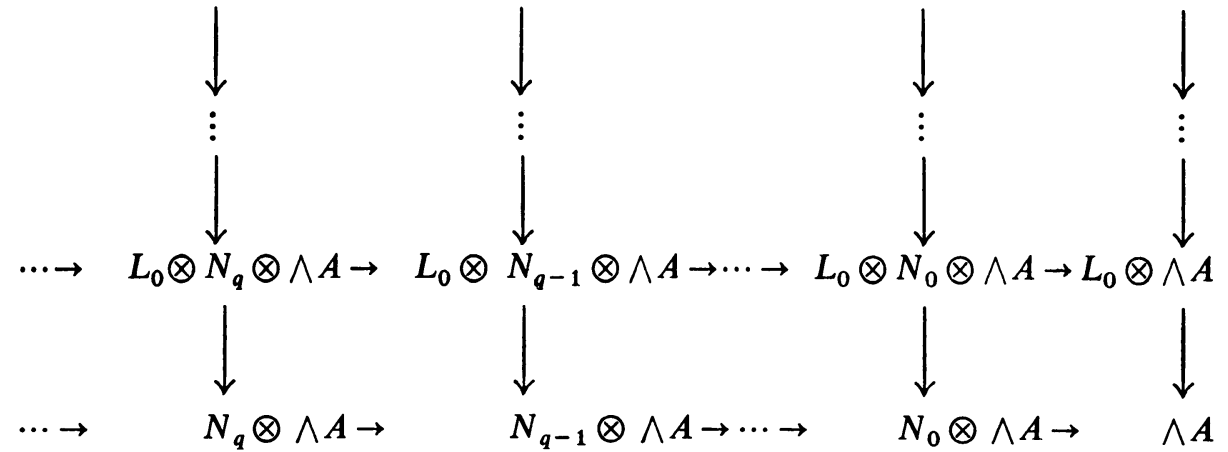

where the maps remain to be defined. 
Since each row of the diagram is simply $L_{p} \otimes \mathfrak{C}(\gamma(f), \wedge A)$ (with the convention that $\left.L_{-1}=R\right)$, the horizontal maps are those induced by the differentiation in $\mathfrak{C}(\gamma(f), \wedge A)$. Similarly, since each column of the diagram (except the one on the extreme right) is $\mathfrak{D}\left(g^{*}, \wedge B^{*}\right) \otimes\left(N_{q}^{\prime} \otimes \wedge A\right)$, we define the vertical maps to be those induced by the differentiation in $\mathfrak{D}\left(g^{*}, \wedge B^{*}\right)$, and the extreme right column, being $\widetilde{C}(\gamma(g f), \wedge A)$, has the obvious differentiation defined on it.

That the diagram thus defined is a double complex is confirmed by the following lemma.

Lemma 4.1. $d d^{\prime}=d^{\prime} d$, where $d$ stands for the horizontal, and $d^{\prime}$ for the vertical maps.

Proof. When $p=q=0$, we have

$$
\begin{aligned}
& d d^{\prime}\left(c_{0} \otimes b_{0} \otimes a\right)=d\left(c_{0} \wedge b_{0} \otimes a\right)=\omega_{c_{0} \wedge b_{0}}(a)=\omega_{c_{0}} \omega_{b_{0}}(a) ; \\
& d^{\prime} d\left(c_{0} \otimes b_{0} \otimes a\right)=d^{\prime}\left(c_{0} \otimes \omega_{b_{0}}(a)\right)=\omega_{c_{0}} \omega_{b_{0}}(a) .
\end{aligned}
$$

If $g=0$, and $p>0$, we have (using the recursive forms of $d$ and $d^{\prime}$ as described in $\$ 1$ )

$$
\begin{aligned}
d d^{\prime}\left(c_{0}\right. & \left.\otimes \cdots \otimes c_{p} \otimes b_{0} \otimes a\right) \\
& =d\left(c_{0} \wedge c_{1} \otimes c_{2} \otimes \cdots \otimes c_{p} \otimes b_{0} \otimes a-c_{0} \otimes d^{\prime}\left(c_{1} \otimes \cdots \otimes c_{p} \otimes b_{0} \otimes a\right)\right. \\
& =c_{0} \wedge c_{1} \otimes c_{2} \otimes \cdots \otimes c_{p} \otimes \omega_{b_{0}}(a)-c_{0} \otimes d d^{\prime}\left(c_{1} \otimes \cdots \otimes c_{p} \otimes b_{0} \otimes a\right) \\
d^{\prime} d\left(c_{0}\right. & \left.\otimes \cdots \otimes c_{p} \otimes b_{0} \otimes a\right) \\
& =d^{\prime}\left(c_{0} \otimes \cdots \otimes c_{p} \otimes \omega_{b_{0}}(a)\right) \\
& =c_{0} \wedge c_{1} \otimes c_{2} \otimes \cdots \otimes c_{p} \otimes \omega_{b_{0}}(a)-c_{0} \otimes d^{\prime}\left(c_{1} \otimes \cdots \otimes c_{p} \otimes \omega_{b_{0}}(a)\right) \\
& =c_{0} \wedge c_{1} \otimes c_{2} \otimes \cdots \otimes c_{p} \otimes \omega_{b_{0}}(a)-c_{0} \otimes d^{\prime} d\left(c_{1} \otimes \cdots \otimes c_{p} \otimes b_{0} \otimes a\right) .
\end{aligned}
$$

By applying induction to the case $q=0$ and arbitrary $p$, we see that we have equality.

If $p=0$ and $q>0$, we have

$$
\begin{aligned}
& d d^{\prime}\left(c_{0} \otimes b_{0} \otimes \cdots \otimes b_{q} \otimes a\right)=d\left(c_{0} \wedge b_{0} \otimes b_{1} \otimes \cdots \otimes b_{q} \otimes a\right) \\
& =c_{0} \wedge b_{0} \wedge b_{1} \otimes b_{2} \otimes \cdots \otimes b_{q} \otimes a-c_{0} \wedge b_{0} \otimes d\left(b_{1} \otimes \cdots \otimes b_{q} \otimes a\right) \\
& \begin{aligned}
d^{\prime} d\left(c_{0} \otimes\right. & \left.b_{0} \otimes \cdots \otimes b_{q} \otimes a\right)=d^{\prime}\left(c_{0} \otimes d\left(b_{0} \otimes \cdots \otimes b_{q} \otimes a\right)\right) \\
& =d^{\prime}\left(c_{0} \otimes\left[b_{0} \wedge b_{1} \otimes b_{2} \otimes \cdots \otimes b_{q} \otimes a-b_{0} \otimes d\left(b_{1} \otimes \cdots \otimes b_{q} \otimes a\right)\right]\right) \\
& =c_{0} \wedge b_{0} \wedge b_{1} \otimes b_{2} \otimes \cdots \otimes b_{q} \otimes a-c_{0} \wedge b_{0} \otimes d\left(b_{1} \otimes \cdots \otimes b_{q} \otimes a\right)
\end{aligned}
\end{aligned}
$$

and thus we have equality. 
We now take care of the remaining cases by induction on $p$, and we may assume that $p$ is positive. Then

$$
\begin{aligned}
& d d^{\prime}\left(c_{0} \otimes \cdots \otimes c_{p} \otimes b_{b_{0}} \otimes \cdots \otimes b_{q} \otimes a\right) \\
& =d\left(c_{0} \wedge c_{1} \otimes c_{2} \otimes \cdots \otimes c_{p} \otimes b_{0} \otimes \cdots \otimes b_{q} \otimes a\right. \\
& \left.-c_{0} \otimes d^{\prime}\left(c_{1} \otimes \cdots \otimes c_{p} \otimes b_{0} \otimes \cdots \otimes b_{q} \otimes a\right)\right) \\
& =c_{0} \wedge c_{1} \otimes c_{2} \otimes \cdots \otimes c_{p} \otimes d\left(b_{0} \otimes \cdots \otimes b_{q} \otimes a\right) \\
& \text { - } c_{0} \otimes d d^{\prime}\left(c_{1} \otimes \cdots \otimes c_{p} \otimes b_{0} \otimes \cdots \otimes b_{q} \otimes a\right) ; \\
& d^{\prime} d\left(c_{0} \otimes \cdots \otimes c_{p} \otimes b_{0} \otimes \cdots \otimes b_{q} \otimes a\right) \\
& =d^{\prime}\left(c_{0} \otimes \cdots \otimes c_{p} \otimes d\left(b_{0} \otimes \cdots \otimes b_{q} \otimes a\right)\right) \\
& =c_{0} \wedge c_{1} \otimes c_{2} \otimes \cdots \otimes c_{p} \otimes d\left(b_{0} \otimes \cdots \otimes b_{q} \otimes a\right) \\
& -c_{0} \otimes d^{\prime}\left(c_{1} \otimes \cdots \otimes c_{p} \otimes d\left(b_{0} \otimes \cdots \otimes b_{q} \otimes a\right)\right) \\
& =c_{0} \wedge c_{1} \otimes c_{2} \otimes \cdots \otimes c_{p} \otimes d\left(b_{0} \otimes \cdots \otimes b_{q} \otimes a\right) \\
& -c_{0} \otimes d^{\prime} d\left(c_{1} \otimes \cdots \otimes c_{p} \otimes b_{0} \otimes \cdots \otimes b_{q} \otimes a\right)
\end{aligned}
$$

and our inductive argument is complete.

What we are ultimately interested in doing is looking at some subcomplexes of this double complex, and then augmenting them. Actually, what we have in mind is the following. We consider maps $R^{m} \stackrel{f}{\rightarrow} R^{n} \stackrel{g}{\rightarrow} R^{r}$, and we want to obtain a double complex which relates $K\left(\Lambda^{p} f\right), K\left(\Lambda^{p} g\right)$ and $K\left(\bigwedge^{p} g f\right)$ for various $p$ 's. We have the chain map of $K\left(\wedge^{p} g f\right)$ into $K\left(\wedge^{p} g\right)$, and we "imbed" this chain mapping in a double complex, the general form of which is the double complex described above. Of course we are already familiar with the fact that the Koszul complex is obtained from a complex of the form $\mathfrak{C}(\gamma, \wedge A)$ by restricting certain indices of summation, and by augmentation. These restrictions on indices pose a rather nasty problem of notation, and to make the reading a little more intelligible, we introduce some simplifying notation.

We return then to the situation $A \stackrel{f}{\rightarrow} B \stackrel{g}{\rightarrow} C$, and consider $\mathfrak{C}\left(\gamma(g f), \wedge^{v} A, u\right), \mathfrak{C}\left(\gamma(g), \wedge^{v} B, u\right)$ and $\mathfrak{C}\left(\gamma(f), \wedge^{v} A, \rho\right)$. We let $\lambda=v+u$ and $\sigma=v+\rho$, and we agree to hold these numbers fixed throughout. We have the chain map of $\mathfrak{C}\left(\gamma(g f), \wedge^{v} A, u\right)$ into $\mathbb{C}\left(\gamma(g), \wedge^{v} B, u\right)$, and we want to imbed this chain map into a double complex involving $\mathfrak{C}\left(\gamma(f), \wedge^{v} A, \rho\right)$.

Using the previously defined double complex as our guide, we first observe that

$$
\mathfrak{C}_{p+1}\left(\gamma(g f), \wedge^{v} A, u\right)=\Sigma \Lambda^{s_{0}+u} C^{*} \otimes \Lambda^{s_{2}} C^{*} \otimes \cdots \otimes \Lambda^{s p} C^{*} \otimes \Lambda^{\lambda+\Sigma s_{i}} A .
$$

This last term may then be written as $\Sigma_{t} L_{p}(t) \otimes \Lambda^{\lambda+t} A$ where 


$$
L_{p}(t)=\Sigma \Lambda^{s_{0}+u} C^{*} \otimes \wedge^{s_{1}} C^{*} \otimes \cdots \otimes \wedge^{s_{p}} C^{*},
$$

the summation running over all $s_{0} \geqq 0, s_{i} \geqq 1$ for $i>0$, and $\sum_{i=0}^{p} s_{i}=t$.

We now consider the following diagram:

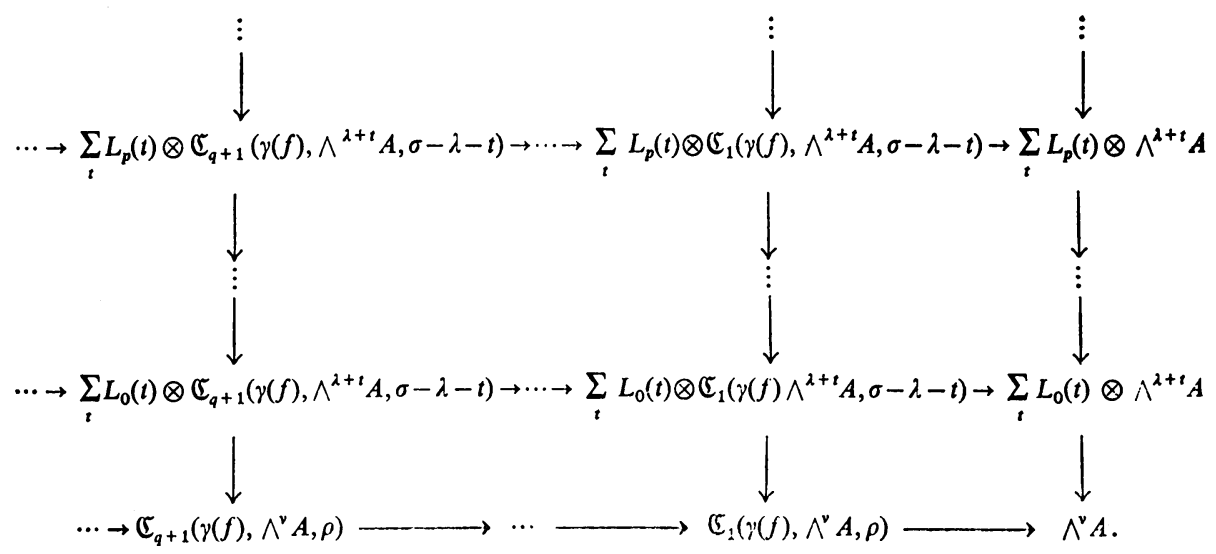

Each row of this diagram is a subcomplex of the corresponding row of the double complex defined before, i.e., it is clear that each module in this diagram is a submodule of the corresponding module in the double complex previously defined, and is stable under the horizontal differentiation there defined. Thus our horizontal maps in this diagram above are simply the restriction of the horizontal differentiation maps defined before. If we now show that the columns of this diagram are stable under the vertical maps of our old double complex, we will have shown that the above diagram is indeed a double complex.

Consider an element in $\Sigma L_{p}(t) \otimes \mathfrak{C}_{q+1}\left(\gamma(f), \wedge^{\lambda+t} A, \sigma-\lambda-t\right)$. We may fix an integer $t$ and simply look at an element in $L_{p}(t) \otimes \mathfrak{C}_{q+1}\left(\gamma(f), \wedge^{\lambda+t} A, \sigma-\lambda-t\right)$. To make it a little easier, let us recall that $\mathfrak{C}_{a+1}\left(\gamma(f), \wedge^{\lambda+t} A, \sigma-\lambda-t\right)$ $=\Sigma \wedge^{u_{0}+\sigma-\lambda-t} B^{*} \otimes \wedge^{u_{1}} B^{*} \otimes \cdots \otimes \wedge^{u_{q}} B^{*} \otimes \wedge^{\sigma+\Sigma u_{i}} A$ where the summation runs over $u_{0} \geqq 0, u_{i} \geqq 1$ for $i \geqq 1$. Thus we may finally take our element $\alpha$ to be of the form

$$
\alpha=c_{0} \otimes c_{1} \otimes \cdots \otimes c_{p} \otimes b_{0} \otimes b_{1} \otimes \cdots \otimes b_{q} \otimes a
$$

where $c_{0} \in \Lambda^{s_{0}+u} C^{*}, c_{i} \in \Lambda^{s_{i}} C^{*}, b_{0} \in \Lambda^{u_{0}+\sigma-\lambda-t} B^{*}, b_{i} \in \Lambda^{u_{i}} B^{*}, a \in \Lambda^{\sigma+\Sigma u_{i}} A$, and $\sum s_{i}=t$. Then

$$
\begin{aligned}
d^{\prime}(\alpha)= & {\left[\sum_{i=0}^{p-1}(-1)^{i} c_{0} \otimes \cdots \otimes c_{i} \wedge c_{i+1} \otimes \cdots \otimes c_{p} \otimes b_{0}+(-1)^{p} c_{0} \otimes \cdots \otimes c_{p-1} \otimes c_{p} \wedge b_{0}\right] } \\
& \otimes b_{1} \otimes \cdots \otimes b_{q} \otimes a
\end{aligned}
$$

and we want to show that this is an element of

$$
\sum_{t} L_{p-1}(t) \otimes \mathbb{C}_{q+1}\left(\gamma(f), \bigwedge^{\lambda+t} A, \sigma-\lambda-t\right)
$$


if $p>0$, otherwise that $d^{\prime}(\alpha) \in \mathfrak{C}_{q+1}\left(\gamma(f), \wedge^{\nu} A, \rho\right)$.

If $p=0$, we have $d^{\prime}(\alpha)=c_{0} \wedge b_{0} \otimes b_{1} \otimes \cdots \otimes b_{q} \otimes a$. But we also have that $s_{0}=t$, so $c_{0} \wedge b_{0} \in \wedge^{u_{0}+\sigma-\lambda+u} B^{*}=\wedge^{u_{0}+\rho} B^{*}$. Thus $d^{\prime}(\alpha) \in \wedge^{u_{0}+\rho} B^{*} \otimes \wedge^{u_{1}} B^{*} \otimes \cdots$ $\otimes \wedge^{u_{q}} B^{*} \otimes \wedge^{\sigma+\Sigma u_{i}} A$ and hence is in $\widetilde{C}_{q+1}\left(\gamma(f), \wedge^{v} A, \rho\right)$.

If $p>0$, it is clear that each of the terms $c_{0} \otimes \cdots \otimes c_{i} \wedge c_{i+1} \otimes \cdots \otimes c_{p} \otimes b_{0} \otimes \cdots$ $\otimes b_{q} \otimes a$ is in the right place, and hence it remains only to check $c_{0} \otimes \cdots$ $\otimes c_{p-1} \otimes c_{p} \wedge b_{0} \otimes \cdots \otimes b_{q} \otimes a$. But we claim that this element is in $L_{p-1}\left(t-s_{p}\right) \otimes \mathbb{C}_{q+1}\left(\gamma(f), \wedge^{\lambda+t-s_{p}} A, \sigma-\lambda-t+s_{p}\right)$ as can be easily checked.

Another way to see that the columns are stable is to observe that $\Sigma_{t} L_{F}(t) \otimes \mathbb{C}_{q+1}\left(\gamma(f), \wedge^{\lambda+t} A, \sigma-\lambda-t\right)$ is isomorphic to $\Sigma_{\tau} D_{p+1}\left(g^{*}, \wedge^{\rho+\tau} B^{*}, u\right)$ $\otimes N_{q}(\tau)$ where $N_{q}(\tau)=\Sigma \wedge^{u_{1}} B^{*} \otimes \cdots \otimes \wedge^{u q} B^{*} \otimes \wedge^{\sigma+\Sigma u_{i}+\tau} A$ with $u_{i} \geqq 1$. This fact will shortly be used, but may be verified fairly straightforwardly.

Now that we have our double complex, we are in a position to augment it. Consider the case, then, when $A=R^{m}, B=R^{n}$, and $C=R^{r}$, i.e., $R^{m} \stackrel{f}{\rightarrow} R^{n} \stackrel{g}{\rightarrow} R^{r}$. We then take $v$ to be any positive integer less than or equal to $r$, we choose $\lambda=r+1$, and $\sigma=n+1$. These are the choices of $\lambda$ and $\sigma$ which lead to the appropriate Koszul complexes.

As mentioned before, we have the chain map $\mathscr{C}\left(\gamma(g f), \wedge^{v} R^{m}, r+1-v\right)$ $\rightarrow \mathfrak{C}\left(\gamma(g), \wedge^{v} R^{n}, r+1-v\right)$. Thus the double complex defined above may be augmented by the addition of an extra column on the extreme right, i.e., by adding on $\mathfrak{C}\left(\gamma(g), \wedge^{v} R^{n}, r+1-v\right)$. By doing this, each row of the complex becomes $\Sigma_{t} L_{p}(t) \otimes K\left(\wedge^{\lambda+t} f\right)$. We can also augment this double complex by adjoining the extra row:

$$
\cdots \rightarrow 0 \rightarrow 0 \rightarrow \cdots \rightarrow 0 \rightarrow \wedge^{v} R^{r} \stackrel{=}{\rightarrow} \wedge^{v} R^{r},
$$

with the map of $\wedge^{v} R^{m} \rightarrow \Lambda^{v} R^{r}$ being $\Lambda^{v} g f$, and that of $\wedge^{v} R^{n} \rightarrow \Lambda^{v} R^{r}$ being $\wedge^{v} g$.

Our newly obtained augmented double complex, which we will call $X(f, g, v)$, has the property that its two right-hand columns are $K\left(\wedge^{v} g f\right)$ and $K\left(\wedge^{v} g\right)$, its rows (except the bottom one) are $\Sigma_{t} L_{p}(t) \otimes K\left(\Lambda^{\lambda+t} f\right)(\lambda=r+1)$, and its columns are $\Sigma_{\tau} \mathfrak{D}\left(g^{*}, \Lambda^{n+1-v+\tau} R^{n *}, r+1-v\right) \otimes N_{q}(\tau)$.

Having defined the double complex $X(f, g, v)$, we return to the main objective of this section.

THEOREM 4.2. Let $R$ be a local ring, let $f: R^{m} \rightarrow R^{n}$ be a map, and $E$ an $R$-module such that $l($ Coker $f \otimes E)<\infty$. Then we have

$$
\left(\begin{array}{l}
n-1 \\
n-p
\end{array}\right) \Delta^{m} P_{f}(v, E)=\chi H_{*}\left(\wedge^{p} f, E\right)
$$

where $\chi H_{*}\left(\Lambda^{p} f, E\right)=\Sigma_{q}(-1)^{q} l\left(H_{q}\left(\Lambda^{p} f, E\right)\right)$.

Proof. From the given map $f: R^{m} \rightarrow R^{n}$, we get the $S\left(R^{n}\right)$-map $S\left(R^{n}\right) \otimes f: S\left(R^{n}\right) \otimes R^{m} \rightarrow S\left(R^{n}\right) \otimes R^{n}$. We also always have the canonical map $\tau^{n}: S\left(R^{n}\right) \otimes R^{n} \rightarrow S\left(R^{n}\right)$. Therefore, from the two maps $S\left(R^{n}\right) \otimes R^{m} \rightarrow S\left(R^{n}\right) \otimes R^{n}$ 
$\rightarrow S\left(R^{n}\right)$, we get the double complex $X=X\left(S\left(R^{n}\right) \otimes f, \tau^{n}, 1\right)$, and we consider $X \otimes E$.

We know that the double complex $X \otimes E$ is of the form

$$
\cdots \rightarrow K\left(\wedge^{3} h\right) \otimes E \rightarrow K\left(\wedge^{2} h\right) \otimes E \rightarrow K(h) \otimes E \rightarrow K(i d) \otimes E
$$

where $h=S\left(R^{n}\right) \otimes f$ (we are using the fact that the double complex becomes quite simple when $r=v=1$ ). Breaking the terms down still further, we see that the complex is of the form

$$
\begin{gathered}
\cdots \rightarrow K\left(\wedge^{3} f\right) \otimes S\left(R^{n}\right) \otimes E \rightarrow K\left(\wedge^{2} f\right) \otimes S\left(R^{n}\right) \otimes E \rightarrow K(f) \otimes S\left(R^{n} \otimes E\right) \\
\rightarrow K(i d) \otimes S\left(R^{n}\right) \otimes E
\end{gathered}
$$

with respect to the horizontal differentiation $d$, and is of the form

$\cdots \sum_{s \geqq 0} \mathfrak{I}\left(\tau^{n *}, \wedge^{s+n} R^{n *} \otimes S\left(R^{n}\right), 1\right) \otimes N_{2}(s) \otimes E$

$$
\begin{aligned}
& \rightarrow \sum_{s \geqq 0} \mathfrak{I}\left(\tau^{n *}, \bigwedge^{s+n} R^{n *} \otimes S\left(R^{n}\right), 1\right) \otimes N_{1}(s) \otimes E \rightarrow K\left(\tau^{n} h\right) \otimes E \\
& \rightarrow K\left(\tau^{n}\right) \otimes E
\end{aligned}
$$

with respect to the vertical differentiation $d^{\prime}$.

We observe here that $K\left(\tau_{n} h\right)=K\left(\tau_{m}\right) \otimes_{S\left(R^{m}\right)} S\left(R^{n}\right)$ as chain complexes, and hence $H_{*}\left(K\left(\tau^{n} h\right) \otimes E\right)=H_{*}\left(\tau^{m}, S\left(R^{n}\right) \otimes E\right)$. On the other hand, $\mathfrak{D}\left(\tau^{n *}, \wedge^{s+n} R^{n *} \otimes S\left(R^{n}\right), 1\right) \approx \mathcal{C}\left(\gamma\left(\tau^{n}\right), \quad \wedge^{-s} R^{n} \otimes S\left(R^{n}\right), 1\right)$ by $1.6, \quad$ and since $H\left(D\left(\tau^{n *}, \wedge^{s+n} R^{n *} \otimes S\left(R^{n}\right), 1\right) \otimes E\right)$ and $H\left(K\left(\tau^{n}\right) \otimes E\right)$ are annihilated by $\operatorname{Im}\left(\tau^{n}\right)$ (being ordinary Koszul complexes) we see that these homology groups must be finitely generated $R$-mcdules. Therefore their $q$ th homogenecus parts must vanish for all sufficiently large $q$, i.e., the $q$ th homogeneous part of the complexes $I)\left(\tau^{n *}, \wedge^{s+n} R^{n *} \otimes S\left(R^{n}\right), 1\right) \otimes E$ and $K\left(\tau^{n}\right) \otimes E$ are acyclic for all sufficiently large $q$.

Now the map $h=S\left(R^{n}\right) \otimes f$ is of homogeneous degree zerc whereas the map $\tau^{m}$ is of homogeneous degree one, and thus the $q$ th homogeneous part $X(q) \otimes E$ of the complex $X \otimes E$ takes the form:

$K\left(\wedge^{m} f\right) \otimes S_{q}\left(R^{n}\right) \otimes E \rightarrow K\left(\wedge^{m-1} f\right) \otimes S_{q+1}\left(R^{n}\right) \otimes E \rightarrow \cdots \rightarrow K\left(\wedge^{m-i} f\right) \otimes S_{q+i}\left(R^{n}\right) \otimes E$

$$
\rightarrow \cdots \rightarrow K(f) \otimes S_{q+m-1}\left(R^{n}\right) \otimes E \rightarrow S_{q+m}\left(R^{n}\right) \otimes E
$$

with respect to the horizontal differentiation. Therefore the Euler-Poincaré characteristic of $X(q) \otimes E$ computed with respect to the horizontal differentiation is

$$
\begin{aligned}
& \sum_{i=1}^{m}(-1)^{i+1} \chi\left(H_{*}\left(\wedge^{i} f, S_{q+m-1}\left(R^{n}\right) \otimes E\right)\right) \\
= & \sum_{i=1}^{m}(-1)^{i+1}\left(\begin{array}{c}
q+m+n-i-1 \\
n-1
\end{array}\right) \chi H \wedge^{i}(f, E) .
\end{aligned}
$$


On the other hand, each column complex of $X(q) \otimes E$ is, with respect to teh vertical differentiation, acyclic (for sufficiently large $q$ ) except the second one from the right, namely, the $q$ th homogeneous part of $K\left(\tau^{n} h\right) \otimes E=K\left(\tau^{m}\right) \otimes S\left(R^{n}\right) \otimes E$. Therefore the Euler-Poincare characteristic of $X(q) \otimes E$ computed with respect to the vertical differentiation is $\Sigma(-1)^{p} l\left(H_{p}\left(\tau^{m}, S\left(R^{n}\right) \otimes E\right)_{q+m-p}\right)$ which is equal to $\Delta^{m} P_{f}(q, E)$ by 3.1 . Therefore we get

$$
\begin{aligned}
\Delta^{m} P_{f}(q, E) & =\Sigma(-1)^{i+1}\left(\begin{array}{c}
q+m+n-i-1 \\
n-1
\end{array}\right) \chi H_{*}\left(\wedge^{i} f, E\right) \\
& =\sum_{i=1}^{m}(-1)^{i+1} \sum_{j=0}^{n-1}\left(\begin{array}{c}
q+m-1 \\
j
\end{array}\right)\left(\begin{array}{c}
n-i \\
n-1-j
\end{array}\right) \chi H_{*}\left(\wedge^{i} f, E\right) \\
& =\sum_{j=0}^{n-1}\left(\begin{array}{c}
q+m-1 \\
j
\end{array}\right) \sum_{i=1}^{m}(-1)^{i+1}\left(\begin{array}{c}
n-i \\
n-1-j
\end{array}\right) \chi H_{*}\left(\wedge^{i} f, E\right)=\sum_{j=0}^{n-1} a_{j}\left(\begin{array}{c}
q+m-1 \\
j
\end{array}\right)
\end{aligned}
$$

where

$$
\begin{aligned}
a_{j} & =\sum_{i=1}^{m}(-1)^{i+1}\left(\begin{array}{c}
n-i \\
n-1-j
\end{array}\right) \chi H_{*}\left(\bigwedge^{i} f, E\right)=\sum_{k=0}^{m-1}(-1)^{k}\left(\begin{array}{l}
n-1-k \\
n-1-j
\end{array}\right) \chi H_{*}\left(\bigwedge^{k+1} f, E\right) \\
& =\sum_{k=0}^{j}(-1)^{k}\left(\begin{array}{l}
n-1-k \\
n-1-j
\end{array}\right) \chi H_{*}\left(\bigwedge^{k+1} f, E\right) .
\end{aligned}
$$

However, the left-hand side, $\Delta^{m} P_{f}(q, E)$, must be a constant independent of $q$ (by 3.6), and hence we get $\Delta^{m} P_{f}(q, E)=a_{0}=\chi H_{*}(f, E)$, and $a_{j}=0$ for all $j>0$. Thus there remains to be shown that

$$
\chi H_{*}\left(\wedge^{j} f, E\right)=\left(\begin{array}{l}
n-1 \\
n-j
\end{array}\right) \chi H^{*}(f, E) .
$$

We proceed by induction on $j$, the case $j=1$ being trivial.

Assume that

Then we have

$$
\chi H_{*}\left(\wedge^{k} f, E\right)=\left(\begin{array}{l}
n-1 \\
n-k
\end{array}\right) \chi H_{*}(f, E) \quad \text { for } k=1,2, \cdots j .
$$

$$
\begin{aligned}
0=a_{j} & =\sum_{k=0}^{j}(-1)^{k}\left(\begin{array}{l}
n-1-k \\
n-1-j
\end{array}\right) \chi H_{*}\left(\wedge^{k+1} f, E\right) \\
& =\sum_{k=0}^{j=1}(-1)^{k}\left(\begin{array}{l}
n-1-k \\
n-1-j
\end{array}\right)\left(\begin{array}{c}
n-1 \\
n-1-k
\end{array}\right) \chi H_{*}(f, E)+(-1)^{j} \chi H_{*}\left(\wedge^{j+1} f, E\right) \\
& =\sum_{k=0}^{j=1}(-1)^{k}\left(\begin{array}{l}
j \\
k
\end{array}\right)\left(\begin{array}{c}
n-1 \\
j
\end{array}\right) \chi H_{*}(f, E)+(-1)^{j} \chi H_{*}\left(\wedge^{j+1} f, E\right) \\
& =(-1)^{j+1}\left(\begin{array}{c}
n-1 \\
j
\end{array}\right) \chi H_{*}(f, E)+(-1)^{j} \chi H_{*}\left(\wedge^{j+1} f, E\right),
\end{aligned}
$$


and hence

$$
\chi H_{*}\left(\wedge^{i+1} f, E\right)=\left(\begin{array}{c}
n-1 \\
j
\end{array}\right) \chi H_{*}(f, E)=\left(\begin{array}{c}
n-1 \\
n-(j+1)
\end{array}\right) \chi H_{*}(f, E) .
$$

This completes the proof.

COROLlaRY 4.3. Let $R$ be a local ring, $f: R^{m} \rightarrow R^{n}$ a parameter matrix for $R$, and $M=\operatorname{Coker} f$. Then

for all R-modules $E$.

$$
\chi H_{*}\left(\wedge^{p} f, E\right)=\left(\begin{array}{l}
n-1 \\
n-p
\end{array}\right) e_{E}(M)
$$

COROLlARY 4.4. Let $R$ be a local ring, $f: R^{m} \rightarrow R^{n}$ a map, and $E$ an $R$-module such that $l($ Coker $f \otimes E)<\infty$. Then $\chi H_{*}\left(\wedge^{p} f, E\right)$ is a non-negative integer and $\chi H_{*}\left(\wedge^{p} f, E\right)=0$ if and only if $m-n+1>\operatorname{dim} E(1 \leqq p \leqq n)$.

COROLlary 4.5. For a local ring $R(\operatorname{dim} R>0)$, the following statements are equivalent:

(1) $R$ is a Cohen-Macaulay ring.

(2) $e(M)=l(M)$ for all $R$-modules $M$ which are given by a parameter matrix for $R$.

(3) $e(R / \mathfrak{q})=l(R / \mathfrak{q})$ for an ideal of definition $q$ generated by a system of parameters.

We close this section with some remarks involving a few informal computations.

Let $R$ be a local ring, and $\mathfrak{q}$ an ideal of definition of $R$, whose multiplicity is equal to $e$. If $P_{q}(x)$ is the characteristic polynomial for $\mathfrak{q}$, then $P_{\mathfrak{p}}(x)=(e / d !) x^{d}+\cdots$ where $d=\operatorname{dim} R$. Thus we see that the multiplicity of $\mathrm{q}^{k}$ for some integer $k$ is simply $k^{d} e$.

Now if $R$ is moreover a Cohen-Macaulay local ring, and $q$ is generated by a system of parameters, then

$$
l\left(\mathfrak{q}^{v} / \mathfrak{q}^{v+1}\right)=\left(\begin{array}{c}
v+d-1 \\
d-1
\end{array}\right) l(R / \mathfrak{q})
$$

(since the associated graded ring of $q$ is a polynomial ring in $d$ variables over $R / \mathfrak{q})$, and hence

$$
l\left(R / \mathfrak{q}^{k}\right)=\sum_{v=0}^{k}\left(\begin{array}{c}
v+d-1 \\
d-1
\end{array}\right) l(R / \mathfrak{q})=\left(\begin{array}{c}
k+d \\
d
\end{array}\right) l(R / \mathfrak{q})
$$

What we are getting at is the following observation. Let $R$ be a Cohen-Macaulay local ring, and $a_{1}, \cdots, a_{d}$ a system of parameters for $R$. Letting $\phi=\left\{a_{1}, \cdots, a_{d}\right\}$, we may construct $\phi^{(n)}: R^{d+n-1} \rightarrow R^{n}$ as described in $\S 3$, and $\phi^{(n)}$ is a parameter matrix for $R$. Let $M=\operatorname{Coker} \phi^{(n)}$. Then since $R$ is Cohen-Macaulay, and $\phi^{(n)}$ is 
a parameter-matrix, we have $e(M)=l(M)=l\left(R / I\left(\phi^{(n)}\right)\right)$. But $I\left(\phi^{(n)}\right)=q^{n}$ where $q$ is the ideal generated by $a_{1}, \cdots, a_{d}$. Thus we see that

$$
e(M)=\left(\begin{array}{c}
n+d \\
d
\end{array}\right) l(R / q)=\left(\begin{array}{c}
n+d \\
d
\end{array}\right) e
$$

(since $e=l(R / \mathfrak{q}))$, and $e(M)$ is not the multiplicity of $I\left(\phi^{(n)}\right)$.

\section{BIBLIOGRA PHY}

1. M. Auslander and D. A. Buchsbaum, Homological dimension in noetherian rings. II, Trans. Amer. Math. Soc. 88 (1958), 194-206.

2. - Codimension and multiplicity, Ann. of Math. (2) 68 (1958), 625-657.

3. D. A. Buchsbaum, A generalized Koszul complex. I, Trans. Amer. Math. Soc. 111 (1964), 183-196.

4. H. Cartan and S. Eilenberg, Homological algebra, Princton Univ. Press, Princeton, N.J., 1956.

5. I. S. Cohen, Unmixed ideals, Algebraic Geometry Conference Notes, mimeographed, Univ. of Chicago, Chicago, Ill., 1949.

6. J. Eagon, Ideals generated by the subdeterminants of a matrix, Ph. D. dissertation, Univ. of Chicago, Chicago, Ill., 1961.

7. A. Grothendieck, Invariants cohomologiques et profondeur, mimeographed notes, Inst. des Hautes Études, Paris, 1961.

8. P. Samuel, La notion de multiplicités en algébre et en géométrie algèbrique, J. Math. Pures Appl. 9 (1951), 159-205, 207-274.

BRANDEIS UNIVERSITY,

Waltham, Massachusetts 